\title{
Kampfmethode Ultimatum. Von disziplinierender Kollegialität zu widerständiger Solidarität - Fallbeispiele aus dem Gesundheitswesen**
}

\section{Zusammenfassung}

Was ist Solidarität im Krankenhaus? Im Alltag äußert sie sich darin, dass Pflegekräfte die eigenen Schutzrechte ignorieren, um Patientinnen und Patienten, aber auch ihre Kolleginnen und Kollegen „,nicht hängen zu lassen“. Der Beitrag handelt von dem Versuch, aus dieser disziplinierenden Form der Kollegialität eine „Kampf-Solidarität“ zu entwickeln: Teams fordern ultimativ Maßnahmen zur Entlastung und drohen damit, andernfalls freiwillige Leistungen wie das Einspringen außerhalb des Dienstplans zu verweigern. Sie nutzen ihre gestiegene Produktionsmacht, da die Abläufe im Krankenhaus regelhaft darauf basieren, dass sich Pflegekräfte über ihre arbeitsvertraglichen Pflichten hinaus engagieren. Der Beitrag untersucht diese neue Kampfform der Ultimaten anhand von sechs Fallstudien, basierend unter anderem auf Experteninterviews mit Pflegekräften, Gewerkschaftssekretär/innen und betrieblichen Interessenvertreter/innen. Theoretisch knüpft er an Solidaritätskonzeptionen von Bayertz (1998) und den Machtressourcenansatz an. Er kommt zu dem Schluss, dass die Ultimaten dazu führen können, die - wie es ein Krankenpfleger und ver.di-Aktivist ausdrückt - im Klinikalltag ,,auf den Kopf gestellte“ Solidarität „,wieder auf die Füße“ (SWB04, 30-32) ${ }^{1}$ zu stellen. Es entwickelt sich eine Kampf-Solidarität, die die Durchsetzungsfähigkeit in Arbeitskämpfen, aber auch die Bereitschaft zu widerständigem Alltagsverhalten stärkt.

Schlagwörter: Solidarität, Machtressourcen, Gesundheitswesen, Arbeitskampf

* Dr. Daniel Behruzi, Wissenschaftlicher Mitarbeiter an der Technischen Universität Darmstadt, Institut für Soziologie, Dolivostr.15, 64293 Darmstadt. E-Mail: behruzi@ifs.tu-darmstadt.de

** Artikel eingegangen: 15.05.2018, revidierte Fassung akzeptiert nach doppelt-blindem Begutachtungsverfahren: 12.09 .2018

1 Die Interview-Codes beginnen mit der Bezeichnung der Region ( $\mathrm{S}=$ Saarland, SW=Baden-Württemberg; $\mathrm{C}=$ Berlin); in der Mitte steht $\mathrm{B}$ für Beschäftigter und V für Gewerkschaftssekretär/in; am Ende sind die Codes durchnummeriert. Die Zahlen nach dem Komma bezeichnen den Absatz des transkribierten Interviews in MaxQDA. 


\title{
The Ultimatum method of industrial action. From disciplinary collegiality to resistant solidarity-case studies in the health care sector
}

\begin{abstract}
What is solidarity in hospitals? In everyday life, it expresses itself in the fact that nursing staff ignore their own protective rights in order not to "let down" patients as well as colleagues. This contribution examines the attempt to develop "employee solidarity" in the face of the disciplinary nature of collegiality: Teams ultimately demand measures for relief or otherwise threaten to refuse to work hours not laid down in the staff roster. Here, the workforces' bargaining power is enhanced by the fact that hospitals regularly depend upon the engagement of nursing staff beyond their contractual obligations. To this end, the article applies Bayertz' (1998) concept of solidarity and the power resources approach. It comes to the conclusion that ultimatums can have the effect-as posited by a nurse and trade union officer-to put solidarity, which is "turned upside down" in everyday life in hospitals, "back on its feet" (SWB04, 30-32). Such a form of solidarity has the advantage that it strengthens the assertiveness of industrial action as well as increases employees' willingness to resist in their everyday working life. The contribution examines this new ultimatum method of industrial action by drawing on research findings taken from six case studies. This involved interviews with nursing staff, trade union secretaries and works council members.
\end{abstract}

Keywords: Solidarity, power resources, health sector, industrial disputes (JEL: I5, J5)

\section{Einleitung $^{2}$}

„Unbefristete Streiks an Uniklinika Essen und Düsseldorf“ (Bibliomed Pflege vom 6. August 2018); „Ver.di droht mit langem Streik an Uniklinik Homburg“ (Kirch, 2018). Das sind zwei aktuelle Schlagzeilen, die noch vor einigen Jahren für unmöglich gehalten worden wären. Sie dokumentieren: Die Industriellen Beziehungen im Gesundheitswesen sind in Bewegung geraten. Bis nach der Jahrhundertwende waren Arbeitskämpfe in dieser Branche de facto nicht existent. Sie beschränkten sich auf eine zumeist symbolische Beteiligung von Krankenhausbeschäftigten an den Tarifrunden des öffentlichen Dienstes, dessen Tarifwerk die Arbeitsbedingungen weit über die kommunalen Krankenhäuser und landeseigenen Unikliniken hinaus prägte. Ein Grund für die weitgehende Absenz von Arbeitskonflikten im Gesundheitswesen lag in dessen Organisations- und Eigentumsverhältnissen: Anders als in der Privatindustrie fällt ein Großteil der Krankenhäuser unter staatliche oder freigemeinnützige Trägerschaften, die keine Profitabsichten verfolgen. In konfessionellen Häusern gelten Tarifverhandlungen und Streikrecht bis heute als mit dem kirchlichen Selbstordnungsrecht unvereinbar (Bingener, 2012). Diese Grundkonstellation besteht trotz der Expansion privater Träger und marktwirtschaftlich getriebener Veränderungen in der Krankenhausfinanzierung (siehe Abschnitt 2.3) fort. Dennoch ist eine zunehmende Konvergenz der Konfliktkonstellationen im Krankenhausbereich mit denen in der Privatwirtschaft festzustellen. Wie ist das zu erklären? Was hat sich geändert? 
Dieser Beitrag ist Teil einer Suchbewegung, die sich den Ursachen für diese Entwicklung annähert. Er hat nicht den Anspruch, eine umfassende Analyse der Verhältnisse und Arbeitsbeziehungen im Gesundheitswesen zu liefern. Viele relevante Aspekte können nicht systematisch aufgearbeitet werden. Das Forschungsinteresse liegt in den neuen Kampfkonstellationen und -methoden, die sich in jüngster Zeit entwickelt haben. Dass Krankenhausbetten und ganze Stationen durch Streiks geschlossen werden müssen - zuletzt beispielsweise an der Uniklinik Essen (Simon, 2018) - ist ein Novum, dessen Hintergründe es zu untersuchen gilt. Der spezielle Fokus dieses Beitrags liegt allerdings auf einer neuartigen Kampfform, die unterhalb der Schwelle formaler Arbeitskämpfe angesiedelt ist - dem sogenannten Ultimatum. Mit dieser Methode wehren sich einzelne Teams gegen Überlastung - zumeist aus der Krankenhauspflege, in Einzelfällen aber auch aus anderen Beschäftigtengruppen. Sie drohen damit, ab einem bestimmten Zeitpunkt Leistungen zu unterlassen, zu denen sie per Arbeitsvertrag nicht verpflichtet sind. So lehnen sie es beispielsweise ab, auBerhalb des Dienstplans einzuspringen, während unbezahlter Pausen zu arbeiten, Überstunden zu machen, zusätzliche Verantwortlichkeiten oder ärztliche Tätigkeiten zu übernehmen. Dies drohen sie für den Fall an, dass die Geschäftsleitung bis zum genannten Datum konkrete Forderungen nicht erfüllt, die zu einer Entlastung führen sollen. Da die Abläufe in den Krankenhäusern aufgrund der dünnen Personaldecke auf solchen freiwilligen Leistungen der Pflegekräfte basieren, übt die Verweigerungsdrohung einen gehörigen Druck auf die Klinikleitung aus. Die Pflegeteams sind so in vielen Fällen in der Lage, Zugeständnisse und Verbesserungen durchzusetzen.

In der Literatur wird vielfach beschrieben, wie der „Pflegeethos“ (Senghaas-Knobloch, 2008) Pflegekräfte dazu bringt, ihre Gesundheit aufs Spiel zu setzen, weil sie ihre Patient/innen nicht im Stich lassen wollen. Der Fokus des vorliegenden Beitrags liegt jedoch auf einem anderen Faktor: der alltäglichen Kollegialität, der „Solidarität“ mit den anderen Mitgliedern des Teams. Sie spielt unseren Erkenntnissen zufolge eine mindestens ebenso wichtige Rolle dabei, dass Beschäftigte ihre Rechte nicht wahrnehmen und „freiwillig“ gesetzliche, einzel- und tarifvertragliche Grenzen überschreiten. Die zentrale These ist, dass diese disziplinierende Kollegialität im Zuge der neuen Kampfmethoden in eine widerständige „Kampf-Solidarität“ transformiert werden kann.

Empirische Basis sind sechs Fallstudien: Untersucht werden jeweils zwei Stationen aus Krankenhäusern im Saarland, im Raum Stuttgart und in Berlin. Diese Regionen wurden auf Grundlage von Vorgesprächen und vorhandenem Feldwissen als diejenigen identifiziert, in denen die Ultimatum-Methode am häufigsten angewandt wird. Die Fallbeispiele wurden mit Blick auf größtmögliche Vergleichbarkeit ausgewählt: Es handelt sich bei allen um große öffentliche Krankenhäuser, in denen ver.di vergleichsweise gut organisiert ist. Andere Fälle, zum Beispiel aus der Altenpflege, wurden aus Gründen der Vergleichbarkeit nicht berücksichtigt.

Die Untersuchung basiert auf einem Dutzend informeller Gespräche sowie 13 leitfadengestützten Interviews mit Expertinnen und Experten, aber auch auf der Auswertung von Flugblättern, Betriebszeitungen, Vereinbarungen, Broschüren und Dokumentationen. Mittels informeller Vorgespräche wurden das Terrain sondiert, die Fallbeispiele ausgewählt und ein Leitfaden entwickelt. In jeder Untersuchungsregion wurden mit jeweils den zuständigen ver.di-Sekretären und an den Ultimaten beteiligten Pflegekräften sowie Betriebs- und 
Personalräten problemzentrierte Experteninterviews (Mayring, 2002, S. 67) geführt. Ebenso mit einer Funktionärin der ver.di-Bundesverwaltung, um eine überregionale Betrachtung der Geschehnisse zu ermöglichen. Die Experteninterviews beschreiben und bewerten Sachverhalte also aus unterschiedlichen Perspektiven. Sie wurden mit Hilfe des Softwareprogramms MaxQDA einer qualitativen Inhaltsanalyse unterzogen, die sich an Mayring (1994; 2002, S. 114-121) sowie den Einführungen von Cropley (2002, S. 127-136) und Lamnek (2005, S. 517-531) orientiert.

Ein Kritikpunkt am Fallstudien-Ansatz ist das mögliche Fehlen externer Validität (Cropley, 2002, S. 96). Um diese zu gewährleisten, sollten die Untersuchungsobjekte so gewählt sein, dass sie ,hinsichtlich einer gleich oder ähnlich strukturierten größeren Menge von Phänomenen als typische Fälle oder besonders prägnante oder aussagefähige Beispiele gelten“ (Hartfiel \& Hillmann, 1972, S. 160). Letzteres gilt auch für die hier versammelten Fallstudien. Sie zielen nicht auf Repräsentativität, sondern sind Teil einer Suchstrategie, die Hinweise auf die Erklärung neuartiger Phänomene liefern soll.

In den Abschnitten 2.1 und 2.2 werden zunächst theoretische Vorüberlegungen zu Solidaritätskonzeptionen und zum Machtressourcenansatz angestellt, die als analytische Folien dienen. In Abschnitt 2.3 werden Prozesse der Ökonomisierung und der Indirekten Steuerung dargestellt, die als Grundlage für die Entstehung neuer Kampfformen gelten können. In Abschnitt 3 folgen die Fallerzählungen. Die Erkenntnisse zu Prozessen und Akteuren werden in Abschnitt 4 verdichtet und systematisch zusammengefasst. In Abschnitt 5 erfolgt eine Diskussion der Ergebnisse, bevor in Abschnitt 6 ein Fazit gezogen wird.

\section{Theoretische Vorüberlegungen}

\subsection{Solidarität}

Schon seit Durkheim (1992 [1893]) spielt das Thema Solidarität in der Soziologie eine zentrale Rolle. Dennoch existiert kein fest umrissenes analytisches Konzept. Vielmehr „,wird in einzelnen Analysen auf sehr unterschiedliche und zum Teil widersprüchliche Solidaritätsbegriffe zurückgegriffen“, wie Tranow (2012, S. 13) in seinem Versuch einer handlungstheoretischen Fundierung des Begriffs betont. Es gilt daher zunächst, den diesem Beitrag zugrundeliegenden Solidaritätsbegriff darzustellen, ohne dabei den Anspruch einer umfassenden Diskussion der Thematik erheben zu wollen.

Bayertz (1998) unterscheidet (neben weiteren) zwischen zwei Typen von Solidarität: „Gemeinschafts-Solidarität“ bezieht sich auf wechselseitige Bindungen und Verpflichtungen innerhalb partikularer Gemeinschaften. „Kampf-Solidarität“ hingegen definiert er als „die Bereitschaft eines Individuums oder einer Gruppe [...], einem anderen Individuum oder einer anderen Gruppe bei der Durchsetzung seiner oder ihrer Rechte zu helfen“ (Bayertz, 1998, S. 49). Sie ist nicht nur, wie auch andere Formen der Solidarität, exklusiv, insofern Individuen oder Gruppen mit anderen Zielen und Interessen ausgeschlossen werden. Sie ist auch konflikthaft, da das gemeinsame Handeln in der Regel erst dadurch notwendig wird, dass ,zur Realisierung der gemeinsamen Ziele und Interessen Widerstände überwunden werden müssen; und zwar vor allem Widerstände anderer Gruppen, die konkurrierende Interessen vertreten“ (Bayertz, 1998, S. 41). 
Historisch erwächst Kampf-Solidarität auf Basis gemeinsamer (Klassen-) Interessen, „,im Wissen um die gleichartige Betroffenheit durch die soziale Konfiguration des Industriekapitalismus“ (Tenfeld, 1998, S. 197). Sie unterscheidet sich von Wohltätigkeit dadurch, dass „eine grundsätzliche Gleichheit zwischen den Beteiligten existiert, die zu der gegenseitigen Erwartung von Hilfe im Bedarfsfall berechtigt“ (Bayertz, 1998, S. 41, Hervorhebung im Original), sie ist also latent reziprok, basiert aber ,auf Freiwilligkeit, nicht auf Zwang“ (Scherr, 2013, S. 265). Ein bloßes gemeinsames „materielles Interesse“ ist indes nicht hinreichend. Anders als im rational choice Ansatz (Hechter, 1988) besitzt Solidarität für Bayertz auch eine „uneliminierbare moralische Dimension“, da sie der Durchsetzung legitimer, „gerechter Ziele“ dient (Bayertz, 1998, S. 44-45, Hervorhebung im Original). Auch Wildt (1998, S. 211) erklärt mit Bezug auf Honneth (1992) und Van Parijs (1995, 1996), „dass zur Solidarität erstens eine besondere Form von moralischer Motivation oder Altruismus gehört und zweitens ein Bezug auf Kooperation oder jedenfalls auf Wechselseitigkeit".

Mit Tranow (2012, S. 36) gehe ich davon aus, dass soziologisch interessierende Solidarität stets einen Handlungsbezug aufweist und nicht allein Gefühlsregungen beschreibt. Laut Billmann \& Held (2013, S. 24) ist Solidarität allerdings ,nicht nur eine Handlung, sondern auch eine Haltung, und manchmal auch nur ein Gefühl der Verbundenheit“. Sie beruht nicht nur auf Interessen, sondern auch auf Identitäten (Baum, 1975).

„Kampf-Solidarität“ ist von einer im betrieblichen Alltag gelebten Kollegialität zu unterscheiden. Analog zu Thomes (1998) Differenzierung zwischen funktionaler und normativer Integration basiert Alltagskollegialität auf der Notwendigkeit von Kooperation im Arbeitsprozess, also auf „Verschiedenheit“, während „Kampf-Solidarität““ auf „Gemeinsamkeiten" fußt - in diesem Fall dem kollektiven Ziel besserer Arbeitsbedingungen. Hürtgen (2013) differenziert in Bezug auf Kollegialität zwischen einer „menschlichen“ und einer „leistungsbezogenen“ Dimension. Windisch (2017, S. 141) spricht von „Alltagskooperation“ als der „Art und Weise, wie eine Belegschaft bzw. ein Team die gestellten Arbeitsaufgaben gemeinsam bewältigt und welche Arbeitsstile, Routinen, Umgangsformen und Stimmungen dabei vorherrschen [...]. Dabei entwickelt sich eine eingespielte Vertrautheit zwischen den Kolleg/innen und ein Gespür für die Stimmungslage der anderen.“

Diese Alltagskooperation spielt in Krankenhäusern wie in vielen anderen Betrieben eine große Rolle. Sie enthält Elemente von Solidarität, wie die latente Reziprozität und den moralisch verpflichtenden Charakter kollegialen Verhaltens. Von der „Kampf-Solidarität“ unterscheidet sie sich jedoch dadurch, dass die für diese postulierte „Zweipoligkeit“ (Bayertz, 1998, S. 41) fehlt: Laut Bayertz ist „Kampf-Solidarität“ zum einen durch den positiven Bezug auf bestimmte Ziele und Interessen und zum anderen durch das „,charakteristische Engagement gegen einen Gegner“ als „,negativem Bezugspunkt“ gekennzeichnet. Im betrieblichen Kontext ist das in der Regel das Management bzw. der Arbeitgeber. Im Krankenhausalltag geht es hingegen um die kooperative Bewältigung gemeinsamer Aufgaben wie der Patientenversorgung. Wenn sich Beschäftigte in diesem Rahmen füreinander einsetzen und sich kollegial verhalten, ist das nicht mit Solidarität gleichzusetzen. Ich bezeichne dieses Phänomen stattdessen als Kollegialität.

Die Entstehung von Solidarität unter den Beschäftigten wird laut Kröll (2013) durch den Strukturwandel unter neoliberalen Vorzeichen seit Mitte der 1970er-Jahre erschwert. 
Auch Scherr (2013) betont, dass die Solidarisierung von Beschäftigten im postmodernen Kapitalismus voraussetzungsvoll ist. Das gilt umso mehr für die Krankenhäuser, wo zusätzliche Hürden zu überwinden sind. So stehen Arbeitskämpfe in diesem Bereich, die notwendigerweise die Versorgung der Patientinnen und Patienten beeinträchtigen, unter besonderem Rechtfertigungsdruck. Beschäftigte im Gesundheitswesen müssen noch stärker als ihre Kolleg/innen in der Privatindustrie darstellen, dass ihre Ziele ,unter dem Gesichtspunkt der Gerechtigkeit als legitim gelten können“ (Bayertz, 1998, S. 44-45). Und sie müssen die Gefährdung von Menschen ausschließen, zum Beispiel durch sogenannte Notdienst-Vereinbarungen mit dem Arbeitgeber.

Hinzu kommt ein unter Begriffen wie „Zuneigungsgefangenschaft“ (Folbre, 2001) und „Pflegeethos“ (Senghaas-Knobloch, 2008) diskutiertes Dilemma von Beschäftigten im Sozial- und Gesundheitswesen: „Häufig haben sie zu den Kranken [...] persönliche Bindungen aufgebaut. Pflichtbewusstsein und Solidarität ihnen gegenüber führen dazu, dass die Beschäftigten trotz Personalkürzungen und anderer Kostensenkungsmaßnahmen eine gute Versorgung sicherstellen müssen und wollen. Verhandlungen mit Arbeitgebern über Löhne und Arbeitsbedingungen gestalten sich deshalb schwierig und die langfristigen Beziehungen erschweren es, zur Not die Reißleine zu ziehen und die Arbeit niederzulegen oder sich einen neuen Job zu suchen“ (Hipp \& Kelle, 2015, S. 34).

Bischoff (1992, S. 168) kritisierte das „typische Denken“ in der weiblich geprägten Krankenpflege aus feministischer Sicht bereits Anfang der 1990er-Jahre so: „Nicht die Strukturen werden verändert, sondern die einzelne Schwester soll die Strukturen kompensieren. Woher die Probleme kommen, wo die Ursachen der Inhumanität liegen, ob man sie lieber verhütet als ausgleicht, ist für eine solche Krankenpflege keine Frage. Die Gesellschaft wird akzeptiert, wie sie ist: sie ist ja auch gar nicht so wichtig, denn auf den Einzelnen kommt es an, auf die ewige Ich-Du-Beziehung. “

Die meisten Autor/innen kommen aufgrund solcher Rollen(selbst)-Zuschreibungen in Bezug auf die kollektive Interessenartikulation von Pflegekräften zu pessimistischen Perspektiven. So vermutet beispielsweise Rieder (1999, S. 119), „dass es für Krankenschwestern schwierig sein wird, ihre berufliche Identität jenseits der beschriebenen ,Kippbilder (liebend versus herzlos und mütterlich versus genusssüchtig) auszubilden. Dies würde eine Überschreitung oder das Ignorieren der angelegten Identitätsangebote voraussetzen.“

Doch es ist nicht allein die Einstellung zur „Pflege als Berufung“(Voges, 2002), die Loyalität zu den Patientinnen und Patienten, die Pflegekräfte dazu bringt, ihre eigenen Rechte und Arbeitsschutzgesetze zu ignorieren (Becker, 2016). Es ist auch und vor allem die Loyalität zu den Kolleginnen und Kollegen, die Pflegekräfte regelmäßig dazu veranlasst, außerhalb des Dienstplans einzuspringen, Überstunden zu leisten oder gesetzlich vorgeschriebene Pausenzeiten nicht einzuhalten. Der vorliegende Beitrag beschäftigt sich mit Letzterem.

\subsection{Machtressourcen}

Ein theoretischer Bezugspunkt des vorliegenden Beitrags ist der Machtressourcenansatz, der nach den Quellen von Arbeitermacht fragt, wobei der Begriff Arbeiter im Sinne von Lohn- und Gehaltsabhängige verstanden wird. Er „unterstellt ein Interesse mehr oder minder heterogener Arbeiter- und Angestelltengruppen, Asymmetrien in den Austauschbezie- 
hungen von Kapital und Arbeit durch kollektive Mobilisierungen besonderer Machtressourcen zu korrigieren“" (Dörre, 2011, S. 276). Wright (2000, S. 962) und Silver (2005, S. 3044) unterscheiden zwischen struktureller Macht (structural power) und Organisationsmacht (associational power). Erstere erwächst aus der Stellung der Beschäftigten(gruppen) im ökonomischen System und wird in zwei Unterformen unterteilt: Marktmacht (marketplace bargaining power) und Produktionsmacht (workplace bargaining power).

Marktmacht ist primäre Verhandlungsmacht und kann aus verschiedenen Gründen entstehen, zum Beispiel dadurch, dass die Betreffenden über seltene oder besonders nachgefragte Qualifikationen verfügen. Auch ein Fachkräftemangel - wie er für die Gesundheitsberufe und speziell für die Pflege konstatiert wird (SpiegelOnline, 2018) - vermindert die Ersetzbarkeit von Lohnabhängigen und erhöht damit ihre Marktmacht. Der Begriff der Produktionsmacht bezieht sich auf den Produktionsprozess, kann aber auch auf Tätigkeiten im Bereich der Reproduktion bezogen werden. Es ist bemerkenswert, dass es zuletzt insbesondere in Bereichen der Reproduktion - inklusive der Krankenhäuser - zu größeren Arbeitskonflikten gekommen ist (Artus, Birke, Kerber-Clasen \& Menz, 2017). Der Jenaer Arbeitskreis Strategic Unionism (2013, S. 349) führt das unter anderem darauf zurück, dass Reproduktionsarbeit zunehmend warenförmig organisiert ist: „Aus der Kommodifizierung dieser Tätigkeiten erwächst den (oftmals weiblichen) Beschäftigten Reproduktionsmacht. Deren Anwendung in Streiks [...] greift in den Reproduktionsprozess der Ware Arbeitskraft ein.“

Arbeitskonflikte gehen in der Regel mit einem Anwachsen von Organisationsmacht einher. Entscheidendes Kriterium zur Messung von Organisationsmacht ist die „Macht der Zahl“ (Marx, 1972 [1867], S. 196), also der Grad gewerkschaftlicher Organisierung. Allerdings lässt sich die Organisationsmacht nicht allein aus der Zahl der Mitglieder bestimmen, schon allein, weil es sich um eine „rein formale Kategorie“ handelt, die als solche „nichts über die Intensität der Bindung der Mitglieder an die Organisation“ aussagt. „Der Organisationsgrad misst ausschließlich die Fähigkeit einer Organisation zur Beschaffung formalisierter Unterstützungsverpflichtungen“" (Streeck, 1979, S. 72, Hervorhebung im Original). Die Größe von Organisationsmacht hängt ebenso von der Mobilisierungsfähigkeit ab (Müller-Jentsch, 1997, S. 119). Auch wenn diese überwiegend latent bleibt (Dörre, 2011, S. 275), so muss die Fähigkeit zur Mobilisierung doch vorhanden sein - zumindest in den Augen des Verhandlungspartners.

Eine weitere Machtressource ist die institutionelle Macht, die Visser bereits in den 1990er-Jahren konzipiert und in den Gewerkschaftsdiskurs eingeführt hat (Visser, 1995; Ebbinghaus \& Visser, 1999). Als ,sekundäre Machtform“ (Brinkmann \& Nachtwey, 2010, S. 21) entsteht institutionelle Macht ,als Resultat von Aushandlungen und Konflikten, die auf struktureller Macht und Organisationsmacht beruhen. Ihre Besonderheit wurzelt in dem Faktum, dass Institutionen soziale Basiskompromisse über ökonomische Konjunkturen und kurzzeitige Veränderungen gesellschaftlicher Kräfteverhältnisse hinweg festschreiben und teilweise gesetzlich fixieren können. [...] Auf diese Weise präformiert institutionelle Macht die Aushandlungsprozeduren und Handlungsstrategien von kollektiven Akteuren wie Betriebsräten und Gewerkschaften“ (Brinkmann, Choi, Detje, Dörre, Holst, Karakayali \& Schmalstieg, 2008, S. 25).

Zusätzlich zu den genannten Machtquellen konzeptualisiert der Arbeitskreis Strategic Unionism (2013) die gesellschaftliche Macht, die er in Kooperationsmacht und Diskurs- 
macht unterteilt. „Kooperationsmacht meint vor allem, über Netzwerke zu anderen gesellschaftlichen Akteuren zu verfügen und diese für Mobilisierungen und Kampagnen aktivieren bzw. sich an deren Aktionen beteiligen zu können“ (Arbeitskreis Strategic Unionism, 2013, S. 360). Bei der diskursiven Macht, die auf Haug (2009) zurückgeht, kommt hingegen ,zum Ausdruck, erfolgreich in öffentliche Debatten bzw. historisch gegebene hegemoniale Grundstrukturen von Öffentlichkeit intervenieren zu können“(Urban, 2010, S. 444).

Der öffentliche Druck - der ,,vor allem über die Skandalisierung von Ungerechtigkeiten“ (Arbeitskreis Strategic Unionism, 2013, S. 361) entwickelt wird - spielt gerade bei Arbeitskonflikten in Bereichen der öffentlichen Daseinsvorsorge eine große Rolle. Wie bereits erwähnt stehen Arbeitskämpfe in Krankenhäusern unter einem besonderen Rechtfertigungsdruck, da sie die Patientenversorgung einschränken und potenziell gefährden. Zudem ist die direkte ökonomische Wirkung, die Aktionen von Krankenhausbeschäftigten entfalten können, geringer als im Produktionssektor. Die Gewinnung der öffentlichen Meinung, also die Entwicklung von Diskursmacht, ist daher ein bedeutender - wenn nicht der entscheidende - Erfolgsfaktor für Arbeitskämpfe in diesem Bereich. Das wird bei der Analyse der Fallstudien zu berücksichtigen sein.

Macht ist nur als relationale Größe zu begreifen. Wie groß die Macht von Beschäftigten ist, hängt also nicht nur von den ihnen selbst zur Verfügung stehenden Machtquellen ab, sondern auch von den Ressourcen ihres Gegenübers (Brookes, 2018; Schmalz, Ludwig \& Webster 2018). Grundsätzlich ließe sich gegen die Applikation des Machtressourcenansatzes für den vorliegenden Beitrag einwenden, dass dieser bislang eher auf die Verhältnisse in kapitalistischen Wirtschaftsbetrieben gemünzt $\mathrm{zu}$ sein scheint als auf einen weiterhin größtenteils öffentlich oder freigemeinnützig organisierten Bereich der Daseinsvorsorge. Traditionell sind die Arbeitsbeziehungen im Krankenhaus weniger konfliktär und antagonistisch geprägt. Doch die Verhältnisse ändern sich und gleichen sich zusehends an diejenigen in der Industrie an. Der Preiswettbewerb zwingt Klinikleitungen - unabhängig von der Trägerschaft - zu permanenten Rationalisierungsanstrengungen, die wegen des hohen Personalkostenanteils vor allem die Beschäftigten treffen. Klinikleitungen agieren gegenüber Gewerkschaften und Betriebsräten zunehmend wie „normale“ Arbeitgeber und machen auch vor Methoden des „Union Busting“ (Behrens \& Dribbusch, 2014) nicht halt. Das gilt insbesondere für private Betreiber (ver.di, 2018a), doch selbst die landeseigenen Universitätskliniken in Baden-Württemberg bedienten sich Ende 2017 einer einschlägigen Kanzlei, um Streiks zu unterbinden (ver.di, 2017e). Auch in den Krankenhäusern geht es daher zunehmend um machtpolitische Aushandlungsprozesse, die mit dem Machtressourcenansatz untersucht werden können. Allerdings sind nicht alle Machtquellen im untersuchten Feld gleichermaßen relevant, weshalb die gewonnenen Erkenntnisse im Fazit nur in Bezug auf einzelne Aspekte des Ansatzes diskutiert werden.

\section{3 Ökonomisierung und Indirekte Steuerung}

Das Gesundheitsstrukturgesetz von 1993 leitete einen Prozess der Ökonomisierung des Krankenhauswesens ein (Simon, 2016). Zehn Jahre später wurde das Finanzierungssystem grundlegend umgestellt. Das seit 1972 über Jahrzehnte geltende Prinzip der Selbstkostendeckung - die Betriebsausgaben der Krankenhäuser wurden von den Versicherungen unter Maßgabe wirtschaftlichen Handelns vollständig refinanziert - wurde durch ein System von 
Festpreisen (Diagnosis Related Groups, DRG) abgelöst (Rosenbrock \& Gerlinger, 2014). Dieses hat eine „neue Herrschaft der Zahlen“ (Marrs, 2007) im Krankenhaus etabliert.

Das ging mit einer deutlichen Ausweitung des Anteils privater Träger am Krankenhausmarkt einher. So hat sich die Zahl der von Privatkliniken betriebenen Betten seit der Jahrhundertwende auf rund 93.000 (2016) in etwa verdoppelt. Ihre Kapazitäten sind damit zwar immer noch deutlich geringer als die öffentlicher und freigemeinnütziger Betreiber, die Reichweite und der Marktanteil privater Konzerne wächst aber kontinuierlich (Statistisches Bundesamt 2018, S. 15). Diese Entwicklung lässt sich als Verschiebung von Marktgrenzen fassen. Auf Mesoebene, also bezogen auf die Branche insgesamt, werden Marktgrenzen durch Preiswettbewerb und Privatisierung ,auf bislang dekommodifiziertes Terrain“ (Brinkmann, 2011, S. 43) verschoben. Auf betrieblicher Mikroebene findet dies seine Entsprechung in veränderten Formen der Unternehmenssteuerung, die alle Bereiche und Beschäftigten unmittelbar dem Druck des Marktes aussetzen.

Denn die Standardisierung von Krankenhausleistungen ermöglicht es, Kliniken mittels betriebswirtschaftlicher Benchmarks zu vergleichen und in Konkurrenz zueinander zu setzen (Vogd, 2006). Die DRG-Werte richten sich nach Durchschnittskosten, was dazu führt, „dass die Krankenhäuser ihre realen Fallkosten aufgrund eines stipulierten Produktivitätsfortschritts fortwährend absenken und damit die Voraussetzung für eine kostenneutrale Ausweitung der Behandlungstätigkeit schaffen“ (Bode, 2010, S. 194). Dieser „Kellertreppeneffekt“" (Simon, 2013, S. 1785) übt einen permanenten Rationalisierungsdruck aus, auf den die Krankenhäuser in den vergangenen Jahren mit Personalabbau - insbesondere im Bereich der (nicht oder kaum erlösrelevanten) Pflege und der Servicetätigkeiten - sowie mit einer Ausweitung der Fallzahlen reagiert haben. Zugleich haben sich die durchschnittlichen Liegezeiten infolge des medizinischen Fortschritts sowie ökonomischer Anreize sukzessive verkürzt und die Fallschwere hat im Durchschnitt zugenommen. All das führt dazu, dass die Belastung der Pflegekräfte in Deutschland auch im internationalen Vergleich extrem hoch ist: Während eine Pflegefachkraft hierzulande für durchschnittlich 13 Patientinnen und Patienten zuständig ist, sind es in den Niederlanden sieben, in den USA 5,3 (Simon \& Mehmecke, 2017).

Vor diesem Hintergrund basiert die Funktionsfähigkeit der Krankenhäuser zunehmend darauf, dass Pflegekräfte und andere Beschäftigte Leistungen über ihre arbeitsvertraglichen Pflichten hinaus erbringen. Laut DGB-Index Gute Arbeit muss nur weniger als ein Drittel der Beschäftigten in der Regel keine Überstunden leisten, zwei von drei Beschäftigten sind dazu also gezwungen (Roth, 2011). Die Gewerkschaft ver.di hat in einer nicht-wissenschaftlichen Erhebung im Mai 2016 ermittelt, dass die Beschäftigten der Krankenhäuser einen „Überstundenberg“ von insgesamt 35,7 Millionen Stunden vor sich her schieben (ver.di, 2016). Laut einer Online-Befragung von gut 3.500 Pflegekräften aus demselben Jahr ist das Einspringen außerhalb des regulären Dienstplans in der Krankenhauspflege allgegenwärtig: Nur vier Prozent der Pflegekräfte geben an, nie in ihrer Freizeit um die kurzfristige Übernahme von Schichten gebeten zu werden (DBfK, 2016). Hinzu kommen veränderte Aufgabenteilungen zwischen den Berufsgruppen im Krankenhaus. „Rationalisierungsstrategien in Form vertikaler Verlagerungen“ (Becker, 2014, S. 45) zielen darauf ab, Tätigkeiten aufzuspalten und auf geringer qualifizierte (und schlechter entlohnte) Beschäftigtengruppen zu übertragen. Examinierte Pflegekräfte geben zwar Tätigkeiten wie die Essensausgabe an 
Service- und Hilfspersonal ab, weshalb manche examinierte Pflegekräfte dies zunächst begrüßen. Die Übernahme ärztlicher Tätigkeiten führt aber dazu, dass sich die Mehrheit durch die veränderte Aufgabenteilung insgesamt stärker belastet fühlt als zuvor (Bräutigam, Evans, Hilbert \& Öz, 2014, S. 52).

Dass Pflegekräfte im Krankenhaus regelmäßig Aufgaben erfüllen, zu denen sie laut Arbeitsvertrag nicht verpflichtet sind, hat aus Sicht von Gewerkschaften und Interessenvertretungen auch einen potenziell positiven Effekt: Mit „Dienst nach Vorschrift“ kann der laufende Betrieb empfindlich beeinträchtigt werden, was effektive Formen von Widerständigkeit unterhalb des Streiks ermöglicht. Inwieweit Pflegekräfte von dieser Möglichkeit Gebrauch machen, ist Gegenstand dieses Beitrags.

Zugleich ist die Ökonomisierung auch ein Hindernis für einzelbetrieblichen Widerstand. Die einzelnen Kliniken stehen unter hohem Konkurrenzdruck, manchen droht die Insolvenz. Die Leitung dieser Kliniken hat daher oft geringe finanzielle Spielräume. Beschäftigte haben Angst, den eigenen Betrieb und Arbeitsplatz durch Aktionen zu gefährden insbesondere indem sie den Ruf des Krankenhauses schädigen könnten. Das ist wohl der Grund dafür, dass sich die Streiks für Entlastung und auch viele der Ultimaten auf große Universitätskliniken konzentrieren, die als weniger insolvenzgefährdet gelten (BV11, 4).

Nicht nur bei der Einführung marktwirtschaftlicher Mechanismen, auch in Bezug auf die Steuerungsformen von Arbeit manifestieren sich aus der Industrie bekannte Entwicklungen zunehmend auch im Gesundheitswesen. Auch hier etabliert sich ,eine weitgehend enthierarchisierte Struktur der Anweisung und Kontrolle, verbunden mit Formen erweiterter Selbstorganisation und Arbeitsautonomie auf der ausführenden Ebene" (Hirsch-Kreinsen, 2010, S. 453). Zwar war die Selbstorganisation der Stationsteams im Krankenhaus immer schon von großer Bedeutung, sie findet heute aber unter anderen, ökonomisierten Rahmenbedingungen statt. Es gelingt dem Management Marrs (2007) zufolge zwar kaum, die Erlös- und Gewinnoptimierung für die Pflegenden orientierungs- und motivationswirksam zu machen, weil deren ethisch-moralische Ansprüche mit der Ökonomisierung in Konflikt geraten. Dennoch wirkt die „Indirekte Steuerung“ (Peters \& Sauer, 2005; Siemens \& Frenzel, 2016) auch hier: Den Stationsteams wird die Verantwortung dafür übertragen, dass die Patientinnen und Patienten rund um die Uhr versorgt werden - unter Rahmenbedingungen, auf welche die Pflegekräfte keinen Einfluss haben. Das trifft insbesondere auf den Personaleinsatz zu. Dieser gilt als unternehmerische Angelegenheit und entzieht sich daher weitgehend der betrieblichen Mitbestimmung. Eine gesetzliche Personalbemessung, auf die sich Beschäftigte und Interessenvertretungen berufen könnten, gibt es mit Ausnahme weniger Krankenhausbereiche nicht.

Die Kombination beider Entwicklungen - der Ökonomisierung und der Etablierung neuer Steuerungsformen - bildet den Ausgangspunkt und Rahmen für die Veränderungen der Industriellen Beziehungen im Gesundheitswesen.

\section{Falldarstellungen}

Die Strategie der Ultimaten ist Teil eines erweiterten Handlungsrepertoires von Gewerkschaften im Krankenhauswesen. Dazu zählen auch die Streiks für mehr Personal und Entlastung, die zuletzt in mehreren Kliniken stattgefunden haben. Dass die Personalbesetzung 
zum Gegenstand von Tarifauseinandersetzungen gemacht wird, war auch innerhalb der Gewerkschaft ver.di nicht unumstritten, kann aber als punktuell erfolgreiche Ausweitung qualitativer Tarifpolitik interpretiert werden, die zugleich den Druck für eine politische Lösung in Form gesetzlicher Personalvorgaben verstärkt. Die Ultimaten sind Teil dieses Prozesses und können als Vorstufe und Vorbereitung größerer Arbeitskämpfe gelten (BV11, 49; SV01, 16).

Schriftliche Darstellungen der Ultimaten finden sich in lokalen bzw. betrieblichen Publikationen der Gewerkschaft sowie in einer Broschüre der ver.di-Bundesverwaltung (ver.di Region Saar-Trier, o.J.; ver.di Berlin-Brandenburg, o.J.; ver.di, 2015). Die Methode kommt vor allem in drei Regionen zur Anwendung: im Saarland, im Raum Stuttgart sowie in Berlin. Das hängt offenbar vor allem mit den dortigen Akteursstrukturen zusammen. Die Strategie wurde quasi zeitgleich von Aktivist/innen im Saarland und in Stuttgart entwickelt, wenn auch mit gewissen Unterschieden. Im Berliner Universitätsklinikum - das bundesweit als Vorreiter und Beispielgeber der Bewegung für Entlastung gilt - wurde der Ansatz adaptiert und ebenfalls in größerem Maßstab angewandt.

Die Verweigerung freiwilliger Leistungen als Kampfmethode unterhalb des Streiks spielte 2017 zwar auch bei von ver.di bundesweit organisierten Aktionstagen gegen Überlastung eine Rolle. In diesem Rahmen weigerten sich Pflegekräfte vorübergehend, außerhalb des Dienstplans einzuspringen, bestanden auf ihren gesetzlich vorgeschriebenen Pausen oder machten „Dienst nach Vorschrift“ in puncto Hygiene (ver.di, 2017a; ver.di, 2017b; ver.di, 2017c). Anders als die Ultimaten haben die Aktionstage aber eher symbolischen Charakter und dienen der gesellschaftlichen Skandalisierung des Personalmangels - was ver.di außerordentlich gut gelingt. Mit den Ultimaten hingegen versuchen einzelne Pflegeteams, den Arbeitgeber zu konkreten Zugeständnissen zu zwingen, indem sie die dauerhafte Verweigerung freiwilliger Leistungen androhen. Der Beitrag konzentriert sich auf diese Aktionsform, da sie sich potenziell stärker auf das Thema Solidarität auswirkt als einmalige Aktionstage.

Als Fallbeispiele für die vorliegende Untersuchung dienen jeweils zwei Stationen aus Krankenhäusern der drei Regionen. Es handelt sich in allen Fällen um große öffentliche Kliniken, zumeist Universitätskliniken, in denen ver.di vergleichsweise gut aufgestellt ist. Auch hier ist allerdings die Mehrheit der Pflegekräfte in der Regel nicht gewerkschaftlich organisiert. Die Ultimaten sind auch eine Reaktion auf diese Schwäche gewerkschaftlicher Organisationsmacht. Sie können von einzelnen, entschlossenen bzw. gut organisierten Teams begonnen werden, ohne dass die Belegschaft in ihrer Breite bereits arbeitskampffähig sein muss.

Um die Vergleichbarkeit zu gewährleisten, wurde darauf verzichtet, Beispiele aus anderen Einrichtungen oder Beschäftigtengruppen einzubeziehen, die es vereinzelt ebenfalls gibt. Beim Fallbeispiel A handelt es sich um die neurologische Station eines saarländischen Krankenhauses mit insgesamt rund 5.000 Beschäftigten. Die Pflegekräfte der Station riefen im Mai 2015 ein Ultimatum aus: „Wir sind in Sorge sowohl hinsichtlich der pflegerischen Versorgung unserer Patienten als auch in Sorge um unsere eigene Gesundheit"“, hieß es darin. Anfang 2014 hätten der Station noch 26,12 Pflegestellen zur Verfügung gestanden, nun nur noch 18,75. In der Folge hätten sich 3.640 Überstunden angehäuft. „Wir wollen drei zusätzliche Kolleg/innen“, hieß es in dem Text weiter. „Wenn wir bis spätestens 1 . Oktober 
2015 keine neuen Kolleginnen bekommen, dann werden wir nicht länger bereit sein, illegale Tätigkeiten auszuführen.“ Keine Pflegekraft werde dann noch in ihrer Freizeit einspringen, „um kurzfristige Personalausfälle und Unterplanung zu kompensieren“ (ver.di SaarTrier, o.J.). Bei Arbeitsüberlastung werde man nur noch eine Notdokumentation vornehmen. Zudem werde man nicht mehr über die gesetzliche Höchstarbeitszeit von zehn Stunden am Tag hinaus arbeiten. Die große Mehrheit des 26-köpfigen Teams unterzeichnete ein „Teamversprechen“, das ab 20 Unterschriften in Kraft treten und nicht veröffentlicht werden sollte.

Die Pflegekräfte dokumentierten das Ultimatum gegenüber Patient/innen und anderen Beschäftigten mit einem von ver.di produzierten Button mit der Aufschrift „NC 02 Neuro? Logisch! Am 1.10. ist Schluss.“ Die Geschäftsleitung sprach in einem Schreiben von „einem klaren Erpressungsversuch“ (ebenda) und lehnte Verbesserungen zunächst kategorisch ab. Sie untersagte den Beschäftigten, die Buttons bei der Arbeit zu tragen. Ver.di reagierte kreativ: Die Gewerkschaft verteilte statt der Buttons einfache Wäscheklammern - nicht nur auf der betreffenden Station, sondern im ganzen Haus. Schon bald war die Wäscheklammer allgegenwärtig. Auch in anderen Krankenhäusern der Region trugen Pflegekräfte diese „Klammer der Solidarität“ (SV01, 36).

Im Team selbst stieg der gewerkschaftliche Organisationsgrad im Verlauf der Auseinandersetzung auf 65 Prozent. Zwei Wochen vor Ablauf des Ultimatums kam es zur Einigung, nachdem sich der gesamte Klinikvorstand mit dem Stationsteam getroffen hatte: Die Geschäftsleitung sagte zu, drei Pflegekräfte zusätzlich einzustellen und die Zahl der Betten um vier zu reduzieren. Zudem sollten ein Springer-Nachtdienst für das Neurologie-Zentrum eingerichtet und der Station ein weiteres Handy sowie ein Laptop zur Verfügung gestellt werden (SB12).

$\mathrm{Zu}$ denjenigen, die durch das Beispiel ermutigt wurden, gehörten die Pflegekräfte einer onkologischen Station des gleichen Krankenhauses, die das Fallbeispiel B darstellt. Sie kündigten im Oktober 2017 an, ab dem 1. Februar 2018 freiwillige Leistungen zu unterlassen, falls nicht drei zusätzliche Vollzeitstellen examinierter Pflegekräfte eingerichtet würden. Den Anstoß dafür gab ausgerechnet der Arbeitgeber: Im Frühjahr hatte ver.di das Klinikum und andere Landeseinrichtungen in Zusammenhang mit der Tarifrunde der Länder zu Warnstreiks aufgerufen. Die Klinikleitung bestand bei den Verhandlungen über eine Notdienstvereinbarung darauf, die onkologische Station im Früh-, Spät- und Nachtdienst mit fünf, vier bzw. zwei examinierten Pflegekräften zu besetzen. „Wir mussten Kolleginnen, die streiken wollten, wieder auf die Station schicken, um die Mindestbesetzung zu erfüllen“ (Supe, 2018, S. 5), so der zuständige ver.di-Sekretär. Doch in den folgenden Monaten wurde diese, von der Klinikleitung selbst ins Spiel gebrachte Mindestbesetzung immer wieder unterschritten. Das veranlasste das Team zum Ultimatum, das von 17 Pflegekräften (bei 18,5 Vollzeitstellen) durch ihre Unterschrift unter das „Teamversprechen“ unterstützt wurde.

Im Rahmen eines „Eskalationsplans“ steigerten sie sukzessive den Druck auf die Klinikleitung: Ab dem 1. Dezember 2017 zählten sie die Tage bis zum Ablauf des Ultimatums mit Aufklebern auf ihren Arbeitskitteln herunter. Kurz vor Weihnachten organisierte ver.di eine Pressekonferenz, die große öffentliche Aufmerksamkeit erregte. Diese wurde noch dadurch gesteigert, dass die Gewerkschaft Anfang Januar 2018 Pflegekräfte im Saarland 
dazu aufrief, sich am 1. Februar zum Noteinsatz auf der Station zu melden. Geplant war zudem eine 24-stündige Mahnwache auf einem öffentlichen Platz. Dazu kam es dann aber nicht mehr, weil die Klinikleitung zuvor Zugeständnisse machte. Die 15 Tage vor Ablauf des Ultimatums erzielte Einigung sah vor, die Zahl der Pflegefachkräfte von 18,5 auf 21 zu steigern und zwei weitere Medizinische Fachangestellte einzustellen. Zudem sollten strukturelle Veränderungen vorgenommen und die Wirkung der Maßnahmen zwei Monate später evaluiert werden. Zu Beginn hätten die Pflegekräfte ihre Angst überwinden müssen, so eine der Beteiligten. Doch das Ultimatum habe das Team „zusammengeschweißt“ (SB02, 11, 49; ver.di Saar-Trier, o.J.).

Die Ultimaten in der Region Stuttgart unterscheiden sich in einigen Aspekten von denen im Saarland. So wenden sich die Pflegekräfte zumeist nicht an eine breitere Öffentlichkeit und an die Medien. So auch in Fallbeispiel $C$, das sich auch in seiner Dramaturgie deutlich von den beiden erstgenannten Fällen unterscheidet. Nachdem sie ihre Überlastung beinahe täglich in sogenannten Überlastungs- oder Gefährdungsanzeigen (ver.di b+b, 2017) dokumentiert hatten, ohne dass die Klinikleitung reagierte, kündigten die Pflegekräfte der Station in einem Brief an die Zentrumsleitung an, ab sofort nicht mehr außerhalb des Dienstplans einzuspringen. Angesichts der extrem hohen Belastung habe es ,eine große Geschlossenheit im Team“ gegeben (SWB06). Das Vorgehen der Pflegekräfte zeigte unmittelbar Wirkung: An einem Wochenende musste die Station kurzfristig geschlossen werden, da kein Nachtdienst zur Verfügung stand. Danach wurde die Bettenkapazität dauerhaft um ein Drittel reduziert, obwohl die Klinikleitung Bettenschließungen zuvor kategorisch ausgeschlossen hatte. Dieser Erfolg habe sich auf das Alltagsbewusstsein positiv ausgewirkt, so eine ver.di-Vertrauensfrau. „Die Kolleginnen jammern nicht mehr nur, sondern setzen sich für ihre Interessen ein“ (ver.di, 2015, S. 30).

Die dauerhafte Sperrung von Betten forderten auch die Pflegekräfte einer Station eines nahegelegenen Krankenhauses im Juni 2015, in Fallbeispiel D. Andernfalls werde man nicht mehr außerhalb des Dienstplans einspringen. Doch anders als in den anderen untersuchten Fällen blieb die Klinikleitung hart. Sie nannte die Forderungen gegenüber der Presse ,absolut unerfüllbar“ und unterstellte ver.di eine aggressive Kampagne, die der Klinik schade (Braun, 2015). Die zuständige Gewerkschaftssekretärin verwies hingegen darauf, dass die Pflegekräfte lediglich angekündigt hätten, eine freiwillige Leistung künftig nicht mehr zu erbringen - ihren Dienstplan erfüllten sie weiterhin. Wenn dies schon zu einem Zusammenbruch des Systems führe, sei das auf ein Organisationsversagen des Arbeitgebers zurückzuführen.

Der Arbeitgeber ließ das Datum verstreichen, ohne auf die Forderung der Pflegekräfte einzugehen. Ein ver.di-Sekretär berichtet: „Im Endeffekt wurden nachher die Zimmer gesperrt und also die Betten gesperrt, aber das Team hat schon richtig viel Druck gekriegt“" (SWV03, 28). Insbesondere die Stationsleitung, die den Zielen des Teams gegenüber positiv eingestellt war, sei massiv unter Druck gesetzt worden: „Die Stationsleitung war völlig fertig mit den Nerven und das hat das Team natürlich mitgekriegt und das ist ein psychischer Druck. Man will die Stationsleitung ja nicht verheizen und fertig machen, sondern mit der arbeiten sie eng zusammen, die mögen sie auch“ (SWV03, 30). Druck gab es auch von den Pflegekräften der Nachbarstation, die einspringen mussten, als das Ultimatum in die Tat umgesetzt wurde: „Insofern wurden halt die Teams gegeneinander ausgespielt und das 
Team, was das Ultimatum umgesetzt hat, hatte einen ganz schlechten Ruf im Haus. [...] Das wirkt bis heute nach [...] und das ist natürlich was, was man in Zukunft tatsächlich verhindern muss, dass man da eher die Solidarität nicht nur im Team hinkriegt, sondern wirklich auch mit den anderen Teams drumherum" (SWV03, 30).

Obwohl die Klinikleitung letztlich nicht umhin kam, Betten zu sperren und damit die Forderung der Pflegekräfte zu erfüllen, führte dieses Ultimatum nicht dazu, andere Teams zu ähnlichen Aktionen zu motivieren - im Gegenteil. „Das war ja auch Sinn und Zweck von der Geschäftsführung, dass dann eben nicht Nachahmerstationen direkt danach kommen, weil niemand will so schlecht dastehen wie dieses Team dann" (SWV03, 32). Die örtlichen ver.di-Aktiven zogen aus dieser Erfahrung die Schlussfolgerung, möglichst nicht mehr mit nur einem Team in eine solche Auseinandersetzung zu gehen.

Am Berliner Uniklinikum kommen Ultimaten, hier „Notrufe“ genannt, seit dem Frühjahr 2013 immer wieder zur Anwendung. Den Anfang machten zwei benachbarte Nephrologie-Stationen, die einen „Notruf“ an die Geschäftsleitung, den Personalrat und die Öffentlichkeit formulierten und die Schließung von jeweils zehn Betten forderten. „Wir haben vorgerechnet, dass der Betrieb ohne ein systematische Überplanung nicht aufrecht zu erhalten ist", berichtet ein Krankenpfleger (CB09). Bei diesem Fallbeispiel E drohten die Pflegekräfte nicht nur damit, außerhalb des Dienstplans nicht mehr einzuspringen, sondern auch, weitere freiwillige Leistungen zu verweigern. Das betraf Tätigkeiten als Hygiene-, Geräte-, Dokumentations- und Arbeitssicherheitsbeauftragte sowie die Praxisanleitung von Auszubildenden. Auch ärztliche Tätigkeiten - vor allem Blutentnahmen sowie die Applikation von Medikamenten und Infusionslösungen - sollten in unterbesetzten Schichten ,zurückdelegiert“ werden. „Die ärztlichen Kollegen sind oft gar nicht mehr in der Lage, Dialysegeräte zu bedienen oder Infusionen richtig zu legen“, erklärt der Kernaktivist der Station. „Daher hat die Verweigerung dieser Arbeiten ein enormes Druckpotenzial.“ Zudem steige das Selbstbewusstsein der Beschäftigten: „Allen wird klar: Ohne die Pflege läuft es nicht.“ Ver.di machte das Ultimatum öffentlich und informierte die Belegschaft mit 3.000 Flugblättern. Die pflegerische Zentrumsleitung verhandelte mit den vom Team delegierten Sprecher/innen und bot für die eine Station zwei, für die andere drei zusätzliche Stellen an. Auf dieser Basis wurde der Konflikt beigelegt (CB09; ver.di Berlin-Brandenburg, o.J.; ver.di, 2015, S. 25-26).

Obwohl die Strategie der Ultimaten am Berliner Großklinikum seit Jahren flächendeckend angewandt wird, mussten die Pflegekräfte ihre Drohungen noch nie umsetzen. Wie im Saarland machte das Management stets zuvor Zugeständnisse. In einigen Fällen wurden die Auseinandersetzungen beigelegt, bevor diese öffentlich wurden. So auch in Fallbeispiel $F$, einer Stroke Unit zur Behandlung von Schlaganfall-Patient/innen. Einige Jahre zuvor wurde dort die Monitorüberwachung eingeführt. Erst waren es vier, dann sechs Monitore, die permanent beobachtet werden mussten. „Diese anspruchsvolle Aufgabe wurde uns zusätzlich auferlegt, ohne dass wir dafür auch nur einen Cent mehr Geld bekommen haben“, sagt eine Krankenpflegerin über den Anlass des Ultimatums. Die 22 Pflegekräfte forderten monatlich 150 Euro mehr Geld. Andernfalls werde das Team Aufgaben als Beauftragte, ärztliche Tätigkeiten und Flex-Dienste künftig ablehnen, hieß es in ihrem „Notruf“. Voraussetzung seien ein „guter Teamzusammenhalt“ und ein hoher gewerkschaftlicher Organisationsgrad gewesen, so eine Aktivistin (CB10, 26). 
Das Management reagierte schnell: Es bot eine Zulage von monatlich 75 Euro an. Obwohl das nur die Hälfte der geforderten Summe war, habe das Team dies ,in einem demokratischen Abstimmungsprozess angenommen“ (CB10, 16), so eine Krankenpflegerin, die die positive Wirkung der „Notrufe“ auf die Solidarität im Team betont: „Teams, die eine solche Notruf-Aktion zusammen durchgestanden haben, sind in der Regel auch im Arbeitskampf eine Bank“ (CB10; ver.di, 2015, S. 28-29).

\section{$4 \quad$ Prozesse und Akteure}

Entwicklung von Kampfsolidarität im Team. Ein Schlüssel bei der Formierung widerständiger Solidarität im Zuge der Ultimaten ist das sogenannte Teamversprechen. Es muss von einem zuvor festgelegten Teil des Teams - in einem Fall sind das mindestens 90 Prozent der Pflegekräfte - unterzeichnet werden. Erst wenn genügend Unterschriften zusammen sind, wird das Ultimatum offiziell verkündet, die Namen werden nicht veröffentlicht. Durch das Teamversprechen wird die, so formuliert es ein Personalratsmitglied, im Klinikalltag ,,auf den Kopf gestellte“ Solidarität ,wieder auf die Füße gestellt“ (SWB04, 30-32). Die Vorzeichen ändern sich: Nun gilt es nicht mehr als solidarisch, außerhalb des Dienstplans einzuspringen, sondern im Gegenteil: Wer dann noch einspringt, „hat in dem Team nichts mehr zu suchen, er stellt sich dann selber außerhalb des Teams“ (SV01, 39). Alle Beteiligten beschreiben dieses formalisierte Versprechen mit seinem „rituellen, symbolischen Charakter" (CB10, 24) als ein zentrales Instrument zur Herstellung widerständiger Solidarität im Team (SV01, 32; BV11, 35-37; SWB04, 34).

Hinzu kommen weitere Erfahrungen, die die Pflegekräfte während des Ultimatums machen. Sie artikulieren sich gegenüber leitenden Vorgesetzten, überwinden Ängste, zum Beispiel im Umgang mit Medien, und treten sichtbar als kollektive Akteure in Erscheinung, beispielsweise durch Anstecker/Aufkleber oder Aktionen in der Öffentlichkeit. All das befördert den Prozess der Entwicklung von Alltagskollegialität zu „Kampf-Solidarität“, der in Abschnitt 5 ausführlicher beschrieben wird (SV01, 9, 35-37; SB02, 91).

Die Rolle anderer Pflegeteams. Die Solidarisierung anderer Stationsteams im Krankenhaus gilt den Beteiligten als entscheidender Erfolgsfaktor für die Ultimaten (SV01, 38). Sie wird dadurch erschwert, dass , die Teams untereinander [...] sich eigentlich gar nicht kennen, sondern sich immer nur auf das eigene Team beziehen“ (SWV03, 32). In einem Fallbeispiel habe der Arbeitgeber dies gezielt ausgenutzt, um „Stimmungen“ gegen das Team der Ultimatum-Station zu erzeugen, was letztlich zum Scheitern des Ultimatums geführt habe (SWV03, 36; SV01, 37, 90-94).

Die Rolle von Ärzt/innen, Patient/innen und anderen Berufsgruppen. Die Ultimaten sind eine Aktionsform der Pflegeteams. Andere Berufsgruppen spielen in der Regel keine Rolle. Relevant ist lediglich, welche Haltung die Ärztinnen und Ärzte einnehmen, mit denen Berührungspunkte und latente Konfliktlinien bestehen - beispielsweise wenn Pflegekräfte ärztliche Tätigkeiten an die Mediziner/innen ,zurückdelegieren“. Die Haltung von Ärzt/innen gegenüber den Aktionen ist den Interviews zufolge unterschiedlich und hängt von der individuellen Einstellung, vor allem aber von der Verortung innerhalb der ärztli- 
chen Hierarchie ab. Während sich die auf der betreffenden Station tätigen Assistenzärzte zumeist solidarisieren, lehnen Ober- und vor allem Chefärzte die Aktionen oft entschieden ab. Die Ärzteorganisation Marburger Bund spielt in den Auseinandersetzungen keine wahrnehmbare Rolle. Bei Streiks des Pflegepersonals hat sie in einigen Fällen ihre Solidarität bekundet (SV01, 98, 105; CB10, 66-74; BV11, 32; SWV03, 50; SWB04, 13-16; MB1). Wenn es von Patientinnen und Patienten Reaktionen gibt, sind diese den Pflegekräften zufolge meistens positiv (SB02, 93; SV01, 114; SWB04, 52).

Die Rolle vorgesetzter Pflegekräfte. Pflegedienstleitungen und -direktoren sind nach Wahrnehmung der beteiligten Pflegekräfte Teil des Managements. Sie gelten als Verhandlungs- und Konfliktpartner, die konfrontativer oder konzilianter auftreten können (SWB04, 38; SWV03, 28-30; SB02, 29-31, 39-43). Ganz anders wahrgenommen wird die Stationsleitung. Sie ist in der Regel gut in das Team integriert, steht aber aufgrund ihrer hervorgehobenen Stellung unter dem Druck, den Betrieb am Laufen zu halten. Wenn sich eine integrierte Stationsleitung gegen das Ultimatum stellt, ist dessen Umsetzung nach Einschätzung der Gewerkschaftsaktiven fast unmöglich. Auf den betreffenden Stationen hätten diese Leitungskräfte die Ultimaten aber zumeist passiv unterstützt und ,weggehört“ (SV01, 135-137; BV11, 8; SWV03, 30; SWB04, 40).

Die Rolle der betrieblichen Interessenvertretung. Betriebs- und Personalräte haben sich zu den untersuchten Ultimaten unterschiedlich verhalten. Während Personalratsmitglieder, die zugleich ver.di-Vertrauensleute waren, in einigen Fällen als „Ideengeber, Koordinatoren, Helfer in Problemsituationen“ (SWB04, 36; SWV03, 70) fungierten, verhielten sich andere passiv (SWV03, 76). Ein ver.di-Sekretär berichtet, das Verhältnis zu den Interessenvertretungen sei „,manchmal schwierig, [...] weil die oftmals abwartend dabeistehen und auch ein Ultimatum als einen persönlichen Angriff empfinden. Es gibt auch sowas wie Eifersucht" (SV01, 91-93).

Die Rolle der Gewerkschaft. Ver.di spielte bei allen untersuchten Ultimaten eine entscheidende Rolle, allerdings nicht immer in Person eines hauptamtlichen Sekretärs. In einigen Krankenhäusern waren es eher betriebliche Vertrauensleute, die dem Team zur Seite standen (SWB04, 54). Im Saarland ist hingegen der zuständige ver.di-Sekretär bei allen U1timaten stark engagiert, verteilt „Schutzbriefe“ und „Notfallkarten“, mit denen er rund um die Uhr erreichbar ist (SV01, 35, 42). „Wichtig ist, immer wieder vor Ort zu sein, die Kollegen nicht allein zu lassen“, betont dieser Hauptamtliche. ,Weil natürlich [...] kommen die Chefs, der Abteilungsleiter, und versuchen dort rein zu brechen. Und natürlich fangen Kollegen an zu zweifeln. Und das muss man im Team immer wieder auffangen, das ist ganz wichtig“" (SV01, 115).

Er betont - ebenso wie alle anderen haupt- und ehrenamtlichen Gewerkschafter/innen, die in die Ultimaten involviert sind - seinen ,Anspruch [...], keine Stellvertreterpolitik zu machen“. Das heiße aber „,nicht, wie manche Kollegen meinen, dass man nicht die Prozesse führen darf. [...] Sondern ganz im Gegenteil. Das verlangt von dir eine viel intensivere Arbeit" (SV01, 128). Es handelt sich also keineswegs um einen führungslosen Prozess, in dem die Pflegekräfte völlig eigenständig agieren. Alle Beteiligten betonen jedoch, dass das Pflegeteam selbst entscheidet, welche Angebote es annimmt oder zurückweist (ver.di, 2015, 8; SV01, 46; ver.di Region Saar-Trier; SWV03, 54-56; SWB04, 8, 36). In einem Fall berichtet 
ein ver.di-Sekretär davon, dass sich das Team trotz seiner Warnungen für eine „Aktionspause“ entschied, nachdem das Management Gespräche angeboten hatte: „Und ich war von ver.di aus bei dem Treff und habe eben gesagt: ,Hier, damit werdet ihr das Ziel nicht erreichen!' [...] So ist es auch gekommen, [...] diese Gespräche haben alle gar nichts gebracht [...]. Und es ist aber leider dann auf ver.di zurückgefallen dort, weil ,mit den ver.diAktionen hat man nichts erreicht' [...]. Das hat für mich die Lernerfahrung, nächstes Mal viel klarer zu sagen: ,Wenn ihr die Entscheidung jetzt trefft, dann müsst ihr mit den Konsequenzen nachher leben [...] und es dann nicht ver.di sozusagen in die Schuhe schieben“" (SWV03, 56).

Öffentlichkeit und Legitimation. Wie stark die Auseinandersetzungen in die Öffentlichkeit getragen werden, ist in den untersuchten Regionen unterschiedlich. Während ver.di im Saarland mit einem „Eskalationsplan“ (SV01, 34) gezielt versucht, sukzessive den öffentlichen Druck auf die Klinikleitung zu steigern, werden die meisten Ultimaten im Stuttgarter Raum nicht in die überbetriebliche Öffentlichkeit getragen. Am Berliner Universitätsklinikum kommt ein abgestuftes Vorgehen zur Anwendung: Zunächst wird versucht, die Klinikleitung innerbetrieblich zu Zugeständnissen zu bewegen, wenn das nicht reicht, werden die Fälle publiziert (SB02, 69-75, 91; SV01, 35, 73-78; CB10, 29-38, 44; SWB04, 8, 46). Doch auch in den Fällen, die nicht veröffentlicht werden, ist die Drohung damit ein wichtiger Faktor für Erfolge. Es ist also von hoher Bedeutung, ob die Pflegekräfte dazu bereit und in der Lage sind, Diskursmacht zu entwickeln. Ver.di betont bei den Streiks und Ultimaten stets, dass es den Beschäftigten nicht nur um ihre eigenen Interessen gehe, sondern auch um das Gemeinwohl. Das kommt auch in den Parolen zum Ausdruck, die die Gewerkschaft häufig verwendet: „Mehr von uns ist besser für alle!“”; „Nicht der Streik gefährdet die Patienten, sondern der Normalzustand!“. Die Arbeitgeber würden die Verantwortung für Einschränkungen bei der Versorgung tragen. Durch die langfristige Ankündigung der Aktionen - ein markanter Unterschied zu Arbeitskämpfen in anderen Branchen, bei denen in der Regel sehr kurzfristig angekündigt wird, welche Bereiche betroffen sind - habe das Management genügend Zeit, sich auf die Situation vorzubereiten und zum Beispiel die Zahl der Neuaufnahmen zu reduzieren. Auf diese Weise versucht die Gewerkschaft, der besonderen ethischen Problematik von Arbeitskämpfen im Krankenhaus zu begegnen und sowohl die Streikbereitschaft als auch die öffentliche Unterstützung zu erhöhen (SV01, 96; ver.di, 2018b; ver.di, 2018c; ver.di Berlin-Brandenburg, 18).

\section{Diskussion}

\subsection{Von disziplinierender Kollegialität im Klinikalltag...}

Die neuen Steuerungsformen von Arbeit im Krankenhaus haben aus Sicht betrieblicher Interessenvertreter/innen eine problematische, zumindest aber ambivalente Wirkung: „Die, sagen wir mal Verflachung der Hierarchie, also [dass es] ganz wenige Häuptlinge praktisch gibt, was ja erst mal klingt wie: ,Ah, erster Erfolg, kann man autonom seine Arbeit selber bestimmen. ' Aber dann im Endeffekt eben bewirkt hat, dass die ganze Verantwortung nach unten verlagert wurde und die Rückübertragung der Verantwortung nicht mehr möglich 
war, weil die da oben so ausgedünnt worden sind, nicht mehr erreichbar waren. Und das bewirkt dann natürlich, dass man [...] auf seine Rechte verzichtet und [...] das alles praktisch selber gewährleisten muss, was läuft [...] - was eigentlich die Aufgabe von Chefs ist" (SWB04, 32).

In dieser Situation wird das Pflege-Team zur „Schicksalsgemeinschaft“, die ,die Situation irgendwie zu bewältigen“ (SWB04, 2) hat: „Die Teams sagen: ,Ja, die Welt ist schlecht drumherum, aber in der schlechten Welt sind wir noch die Besten. ' Ja? ,Wir versuchen das zu regeln. ' Und diese, also positiven Ansätze werden genommen, dass es funktioniert. Damit erreichen die Kollegen natürlich gar nichts. [...] Die einzige Möglichkeit, wie sie sich [...] retten können aus diesem Teufelskreis, ist, indem sie dann krank werden, oder einen Krankenschein machen. Wie auch immer. Weil das das Einzige ist, was akzeptiert wird, [...] innerhalb des Teams“ (SV01, 18-19).

Auf diese Weise wird „,der Teamzusammenhalt zur Selbstausbeutung genutzt [...], oder zur gegenseitigen Selbstausbeutung“ (BV11, 53). Für die Führungskräfte bedeutet diese Form der Steuerung eine Entlastung, denn ,erstens hat man halt nicht so viel Arbeit damit und zweitens zieht man die Kritik nicht auf sich. Und es funktioniert eben auch sehr gut, dass die Leute dann ihren Kolleginnen und Kollegen zu Liebe das dann machen, was sie vielleicht gegenüber einer Vorgesetzten, einem Vorgesetzten irgendwann dann auch mal sagen würden: ,Ne, ich kann jetzt nicht ans Telefon gehen.“" (BV11, 4). Die Beschäftigten stehen hingegen vor einem Dilemma: „Und dann stellt sich halt immer mehr die Frage: [...] Wo mache ich Abstriche? Am Patienten [oder] verzichte hier auf meine Pause und kann vielleicht doch ein bisschen länger machen und springe ich ein im Frei und stoße meiner Familie vor den Kopf oder provoziere ich unter Umständen, dass meine Kollegin statt für zehn Patienten plötzlich für 15 Patienten zuständig ist [...]? Und das ergibt sich dann halt in der alltäglichen Solidarität, um den Beruf irgendwie ausüben zu können, mit Rahmenbedingungen auf die man halt keinen Einfluss hat" (SWB04, 2).

Dass das „Verantwortungsgefühl gegenüber den Patientinnen und Patienten“ (BV11, 10) Pflegekräfte dazu bringt, ihre eigenen Rechte zu ignorieren und ihre Gesundheit aufs Spiel zu setzen, wird in der Literatur breit diskutiert. Dieses Phänomen findet sich auch in den Interviews wieder. Doch eine mindestens ebenso starke Wirkung scheint das Bedürfnis zu haben, „die Kollegen [...] nicht im Stich zu lassen“ (CB10, 5-10). Ein ver.di-Sekretär benennt beide Aspekte als Motivation, außerhalb des Dienstplans einzuspringen: „[Der Pfleger] kommt aus dem Frei, weil er keine andere Lösung sieht, weil er sich erinnert, dass der Kollege auch aus dem Frei gekommen ist als er krank war und [...] weil er natürlich auch konkret den kranken Menschen sieht, den er nicht allein lassen will“ (SV01, 6). Ein anderer Gewerkschafter betont, die Pflegekräfte ,sehen die direkten Kollegen dann vor sich, wie sie dann alleine [...] arbeiten müssen und wissen am eigenen Leib, was das bedeutet" (SWV03, 8).

Hier wird deutlich, dass dieses Verhalten auf Reziprozität beruht: Die Pflegekraft verknüpft ihre Bereitschaft zu zusätzlichem Engagement mit der Erwartung, dass die Kolleg/innen sich ebenso verhalten. Dementsprechend gelten diejenigen, die auf ihrem Recht bestehen, als „egoistisch“ (BV11, 12) und sind ,im Team isoliert“ (SV01, 5) bzw. ,im sozialen Abseits“ (SWV04, 6). So werde „die Solidarität auf den Kopf gestellt“, meint ein Krankenpfleger, ,weil diese Solidarität eben bedeutet, dass man den ganzen Mist mitmacht, 
auf die Rechte verzichtet“ (SWB04, 32, 30). Auch eine hauptamtliche Gewerkschafterin betont: „Sich gegenseitig im Leid beizuspringen und das Leid zu verlängern, ist zumindest eine fragwürdige Form von Solidarität. Weil Solidarität heißt für mich eigentlich, sich gegenseitig dabei beizustehen, für eine Verbesserung zu kämpfen. Also für mich ist Solidarität auch ein Kampfbegriff" (BV11, 16).

Dieses Verständnis von Solidarität als „Kampfsolidarität“ wird in den Interviews von allen Aktivist/innen grundsätzlich geteilt (CB10, 12; SWV03, 10; SWV04, 4). Ein ver.diSekretär argumentiert allerdings, dass die Kollegialität im betrieblichen Alltag „ein solidarisches Element" hat, das die Gewerkschaft „nicht etwa bekämpfen, sondern stabilisieren“ müsse (SV01, 16-17). In der Vergangenheit habe ver.di das nicht gemacht. Mit der Parole „Dein Frei gehört dir" forderte sie Pflegekräfte individuell auf, ihre Rechte wahrzunehmen und nicht mehr außerhalb des Dienstplans einzuspringen - mit geringem Erfolg (SWV03, 12; BV11, 14). Jetzt versuche ver.di, „von dem Individuellen hin zum Kollektiv [zu] kommen“ (SV01, 10): „Ich war Stationsleiter und ver.di hat sich ausgedacht ,Mein Frei gehört mir.' Also [...] stand ich plötzlich da als Stationsleiter und hab gedacht: ,Scheiße! Jetzt kriege ich Schwierigkeiten damit, [...] einen gewerkschaftlichen Auftrag zu erfüllen. Warum ist denn das so schwer?' Weil ich natürlich den Dienstplan selber organisieren musste und bei Ausfällen selbstverständlich auch Leute angerufen habe: ,Du musst jetzt arbeiten kommen. ' $[\ldots]$ Und da ist mir dann eine Idee gekommen, dass das ganze Problem eben gar nicht lösbar ist, indem ich das nur individuell betrachte und dem Mitglied sage: ,Du musst Nein sagen.', sondern ich brauche kollektive Prozesse. Ich muss also das gesamte Team ansprechen“"(SV01, 4-6).

\section{$5.2 \quad \ldots$ zu widerständiger Solidarität}

Aus Sicht der betrieblichen Akteure hat die Ökonomisierung neben den dargestellten problematischen Auswirkungen auch zur Folge, dass Krankenhausbeschäftigte - nicht nur Ärztinnen und Ärzte, sondern auch Pflegekräfte und teilweise sogar Mitarbeiter/innen der Servicebereiche - Zugriff auf zuvor verschlossene Machtquellen erhalten, namentlich Produktionsmacht: „Wenn ich das Krankenhaus zu einer Fabrik mache, dann müssen die Beschäftigten des Krankenhauses auch lernen, sich wie Arbeiter zu benehmen, [...] zu verstehen, dass ihre große Stärke darin besteht, ihre Ware Arbeitskraft $[\ldots]$ verweigern zu können und damit etwas auszulösen - nämlich fehlende Einnahmen. Das stellt eine Macht dar. Diese Macht hatten sie in den früheren Jahren eben so gesehen nicht" (SV01, 139).

Eine Gewerkschaftsfunktionärin betont, die Beschäftigten sollten sich dieser für den Krankenhausbereich neuen Machtressource bewusst werden: „Wenn ein Krankenhaus nach dem Gewinnprinzip letzten Endes funktioniert, oder nach einem Erlösprinzip, und du sorgst dafür, dass die Erlöse wegfallen, dann hat deine Krankenhausleitung ein Problem, dann richtest du ja einen realwirtschaftlichen Schaden an. Das war bei dem Selbstkostendeckungsprinzip nicht so. [...] Das ist vielen in der Belegschaft noch nicht so klar, was sie da eigentlich für ein Machtmittel in der Hand haben“ (BV11, 55).

Ein anderer ver.di-Sekretär argumentiert allerdings, durch die Verweigerung freiwilliger Leistungen auf einzelnen Stationen werde kein ernsthafter ökonomischer Druck erzeugt (SWV03, 64). Das sei erst bei Streiks der Fall, in deren Folge Betten oder ganze Stationen vorübergehend geschlossen werden müssen. Die beiden Aktionsformen - Ultimaten sowie Betten- und Stationsschließungsstreiks - können indes als Teil einer veränderten Gesamt- 
strategie der Gewerkschaft im Gesundheitswesen interpretiert werden. Denn auch bei der neuen Streikform geht es darum, ,kollektive Prozesse in Gang zu setzen“ (SV01, 82): Während ver.di in vergangenen Arbeitskämpfen an einzelne Beschäftigte appellierte, die Arbeit niederzulegen, geht der Appell jetzt an die Teams. Sie sollen sich kollektiv und im Vorfeld darüber verständigen, wie stark sie sich an der Arbeitsniederlegung beteiligen wollen. Wenn beispielsweise die Hälfte der Pflegekräfte in Streik treten will, fordert ver.di den Arbeitgeber auf, jedes zweite Bett in dieser Zeit nicht zu belegen. Zum Teil werden ganze Stationen zum Streik ,,angemeldet“ - so frühzeitig, dass die Klinikleitung sie leer räumen kann. Zuerst entwickelt wurde diese neue Streikform 2011 am Berliner Universitätsklinikum. Sie löst das Dilemma der Pflegekräfte teilweise auf, sich zwischen Streik und Patientenversorgung entscheiden zu müssen. „Denn: Wo keine Patient/innen sind, kann niemand zu Schaden kommen“ (ver.di Berlin-Brandenburg, o.J., S. 13). Mit dem Argument, das Streikrecht der Pflegekräfte könne letztlich nur so ungehindert in Anspruch genommen werden, versucht ver.di, vor Streiks entsprechende Notdienstvereinbarungen mit den Kliniken zu schließen (ver.di Berlin-Brandenburg, o.J., S. 18-20).

Die ver.di-Aktiven sehen die Ultimaten dabei auch als Vorbereitung eines Betten- und Stationsschließungsstreiks. Sie haben ,den Effekt, dass die Beteiligten die Erfahrung machen: Nur wenn man sich gemeinsam wehrt, kann man etwas erreichen. Je mehr Teams diese Erfahrung machen, desto breiter wird die Bewegung für Entlastung und umso mehr können wir erreichen“ (SV01b, 16). Eine Gewerkschafterin betont: „Wenn man Stationen hat, die schon einmal durch so ein Ultimatum gegangen sind, [...] wissen [sie], wie der Teamzusammenhalt funktioniert und wie man den auch in schwierigen Situationen aufrecht erhält und [sind] deswegen wahrscheinlich auch gute Streikstationen, das würde ich auf jeden Fall sagen“ (BV11, 51). Alle Aktivist/innen bestätigen die Einschätzung, dass erfolgreiche Ultimaten die Bereitschaft zu kollektiven Aktionen inklusive Streiks steigert. Das gilt selbst dann, wenn das Ultimatum keinen oder nur einen unzureichenden Erfolg gebracht hat. Die betreffenden Teams seien dennoch zu „Streikteams“ geworden. „Die, die Ultimaten gemacht haben, die einmal so einen kollektiven Prozess durchgemacht haben, die streiken auch“ (SWV03, 40, 70).

Die Ultimaten gehen in der Regel mit einer Zunahme von Organisationsmacht einher. Ein ver.di-Sekretär berichtet von „,enormen Mitgliederzugewinnen“ (SV01, 85) in den beteiligten Teams. Eine Pflegekraft sagt: „Als wir das Teamversprechen oder den Notruf aufgesetzt hatten, waren wir zu 90 Prozent gewerkschaftlich organisiert, weil man das auf jeden Fall durch die Gewerkschaft begleiten lassen muss“ (CB10, 26). Die intensive Begleitung durch die Gewerkschaft ist offenbar in der Tat wichtig für den Erfolg von Ultimaten. Sie stellt „das nötige Know-how“ (SV01, 129) und rechtlichen Schutz zur Verfügung und fängt einen Großteil des Drucks durch den Arbeitgeber auf (SV01, 126-130).

Den Erzählungen zufolge befördert die erlebte „Kampf-Solidarität“ auch nach Ende der Ultimaten widerständiges Verhalten. Eine Krankenpflegerin berichtet, dass ihr Team sich seither ,,selbstbewusster gegenüber dem Arbeitgeber präsentiert und [...] gemeinsam [...] gegen vorherrschende Verhältnisse im Betrieb irgendwie vorgeht. [...] Bei uns war das tatsächlich so gewesen, dass da nicht mehr so irgendwie die üblichen Verdächtigen [...] Missstände angeprangert haben, sondern dass so nach und nach tatsächlich die Kollegen ein Selbstbewusstsein entwickelt haben und auf einmal mehrere Leute im Konflikt sich zu Wort gemeldet 
haben, was früher überhaupt nicht so gewesen ist“" (CB10, 26-28). Ein Personalrat berichtet von derselben Wirkung erfolgreicher Ultimaten: „Das stärkt auf jeden Fall das Team, also den Zusammenhalt. Die spüren auch die Macht, also das ist eindeutig von den Aussagen der Leute, dass sie merken, [...] dass es eine Auswirkung hat, was sie machen“ (SWV04, 24).

Auch auf das Verhalten der Klinikleitung in den alltäglichen Konflikten haben die Aktionen Auswirkungen. Viele berichten davon, dass sich die widerständigen Teams „Respekt verschafft" (SWV04, 10) haben und von den Führungskräften seither ernst genommen werden (SB02, 43; SV01, 64). „Das wirkt alles erst mal: ,Wir sind stark!““ (SV01, 78). Ein Krankenpfleger betont, dass dieser Effekt in der Regel länger anhält: „Das hat ja meistens auch nachhaltige Wirkung, dass wenn man einmal sich Respekt verschafft hat, dann reicht manchmal nur eine Mail, dass dann wieder was passiert, ohne dass wir jetzt einen großen Aufstand machen müssen“ (SWB04, 28).

\section{Fazit}

Die vorliegende Untersuchung behandelt nur einen Aspekt der veränderten Arbeitsbeziehungen im Krankenhaus. Die im Beitrag erwähnte neue Streikstrategie, die die Schließung von Betten und Stationen bewirken kann, wäre ebenfalls eingehender auf ihre Voraussetzungen und Wirkungen hin zu untersuchen. Gleiches gilt für das Zusammenspiel der verschiedenen Ebenen - der betrieblichen, tariflichen und der politischen - die ver.di in der Auseinandersetzung um mehr Personal und Entlastung im Krankenhaus nutzt (ver.di, 2017d). Welche Machtressourcen werden dabei ins Feld geführt? Wie korrelieren die Aktivitäten auf den verschiedenen Ebenen miteinander? Inwiefern ist die Gewerkschaft in der Lage, Marktgrenzen (zurück) zu verschieben? Welche Bündnisse geht sie dabei ein und welche Solidaritätspotenziale kann sie mobilisieren? Wie verlaufen die innergewerkschaftlichen Diskussionen zur strategischen und taktischen Ausrichtung? Welche Rolle spielen andere Akteure wie Staat, Klinikbetreiber und Krankenkassen? All diese Fragen wären eingehendere Studien wert, die den Rahmen dieser Arbeit bei Weitem gesprengt hätten.

Dennoch hat sie interessante Erkenntnisse zutage befördert, auch in Bezug auf Solidaritätskonzeptionen. Die Kollegialität - die im Alltag eine disziplinierende Wirkung entfaltet - wird durch die Ultimaten in eine „Kampf-Solidarität“ verwandelt, die die Durchsetzungsfähigkeit in Arbeitskämpfen, aber auch die Bereitschaft zu widerständigem Alltagsverhalten stärkt. Ihren formalisierten Ausdruck findet diese Solidarität im „Teamversprechen“, das die Pflegekräfte zumeist sogar schriftlich fixieren und unterschreiben. Damit gehen sie eine Art Pakt ein, sich kollektiv anders zu verhalten als im Alltag: Bei Inkrafttreten des Ultimatums gilt es nicht mehr als solidarisch, kurzfristig einzuspringen, sondern im Gegenteil, sich an die kollektiv beschlossene Aktion zu halten. Ob diese Transformation aber tatsächlich von Dauer ist oder - was zu vermuten ist - sich im Alltag wieder zurück entwickelt, kann in der vorliegenden Studie nicht geklärt werden.

In den Termini des Machtressourcenansatzes können die Ultimaten als Nutzung der (gestiegenen) Produktionsmacht von Pflegekräften verstanden werden. Schon die Verweigerung freiwilliger Leistungen birgt das Potenzial, die Abläufe in Krankenhäusern empfindlich zu stören. Werden die Ultimaten in Zusammenhang und als Vorbereitung von Streiks 
verstanden, bei denen es zur vorübergehenden Schließung von Betten und ganzen Stationen kommt, beinhalten sie ein ökonomisches Druckpotenzial, das so früher nicht bestand.

Eine wichtige Rolle in den Auseinandersetzungen um mehr Personal und Entlastung im Krankenhaus spielt zudem die Diskursmacht: Potenziell entscheidender als die Störung der Abläufe und die Verursachung eines betriebswirtschaftlichen Schadens ist der Reputationsverlust für die betreffenden Kliniken, der mit öffentlichkeitswirksamen Aktionen der Beschäftigten einhergehen kann. Die im Wettbewerb stehenden Krankenhäuser sind sehr darauf bedacht, ihren Ruf in der Bevölkerung zu wahren. Das können Beschäftigte bei Ultimaten oder anderen Aktionsformen nutzen, wenngleich die übermäßige mediale Skandalisierung der Zustände in einem konkreten Haus auch als riskant eingeschätzt wird. Eindeutig ist der Befund, dass erfolgreiche Ultimaten zur Steigerung von Organisationsmacht führen - nicht nur quantitativ bezogen auf Mitgliedszahlen, sondern vor allem durch die steigende Mobilisierungsfähigkeit und Eigenaktivität der beteiligten Beschäftigten.

Offen ist, inwieweit die betrieblichen, tarifpolitischen und öffentlichen Auseinandersetzungen in den Krankenhäusern tatsächlich zu einer Rück-Verschiebung der Marktgrenzen führen können. Einerseits ist der Prozess der Etablierung marktwirtschaftlicher Mechanismen im Krankenhauswesen weit fortgeschritten und erscheint nur schwer umkehrbar zu sein. Andererseits haben die gewerkschaftlichen Aktivitäten bereits erstaunliche Auswirkungen auf den gesellschaftlichen und politischen Diskurs. Union und SPD sahen sich dazu veranlasst, in ihrem Koalitionsvertrag nicht nur zu versprechen, „die Arbeitsbedingungen und die Bezahlung in der Alten- und Krankenpflege sofort und spürbar [zu] verbessern“, sondern auch, die „Pflegepersonalkosten besser und unabhängig von Fallpauschalen“ zu vergüten (Koalitionsvertrag, 2018, S. 96-99). Würden die Aufwendungen für das Pflegepersonal dem Preiswettbewerb tatsächlich vollständig entzogen, wäre das in der Tat eine bemerkenswerte Verschiebung von Marktgrenzen, die der „Logik des Marktkapitalismus“ (Aglietta, 2000) entgegenlaufen würde.

\section{Literaturverzeichnis}

Aglietta, M. (2000). Ein neues Akkumulationsregime. Die Regulationstheorie auf dem Prüfstand. Hamburg: VSA.

Arbeitskreis Strategic Unionism (2013). Jenaer Machtressourcenansatz 2.0. In S. Schmalz \& K. Dörre (Hrsg.), Comeback der Gewerkschaften? Machtressourcen, innovative Praktiken, internationale Perspektiven (S. 345-375). Frankfurt/Main: Campus.

Artus, I., Birke, P., Kerber-Clasen, S. \& Menz, W. (Hrsg.). (2017). Sorge-Kämpfe. Auseinandersetzungen um Arbeit in sozialen Dienstleistungen. Hamburg: VSA.

Baum, R. C. (1975). The System of Solidarities. Indian Journal of Sociology, 16/1975, 306-353.

Bayertz, K. (1998). Begriff und Problem der Solidarität. In K. Bayertz (Hrsg.), Solidarität - Begriff und Problem (S. 11-53). Frankfurt/Main: Suhrkamp.

Becker, K. (2014). Von Florence Nightingale zu Adam Smith? zfwu, 15 (1), 33-52. doi: 10.1688/zfwu-2014-01-becker

Becker, K. (2016). Loyale Beschäftigte - ein Auslaufmodell? Pflege \& Gesellschaft, 2/2016, 145161. doi: $10.3262 / \mathrm{P} \& G 1602145$

Behrens, M. \& Dribbusch, H. (2014). Arbeitgebermaßnahmen gegen Betriebsräte: Angriffe auf die betriebliche Mitbestimmung. WSI-Mitteilungen, 2/2014, 140-148.

doi: 10.5771/0342-300X-2014-2-140 
BibliomedPflege (2018). Unbefristete Streiks an Uniklinika Essen und Düsseldorf. Abgerufen am 27. August 2018 von

https://www.bibliomed-pflege.de/alle-news/detailansicht/36031-unbefristete-streiks-anuniklinika-essen-und-duesseldorf/

Billmann, L. \& Held, J. (2013). Einführung. Solidarität, kollektives Handeln und Widerstand. In L. Billmann \& J. Held (Hrsg.). Solidarität in der Krise: Gesellschaftliche, soziale und individuelle Voraussetzungen solidarischer Praxis (S. 13-29). Tübingen: Springer VS.

Bingener, R. (2012). Eine Bestätigung für die Kirchen. Frankfurter Allgemeine Zeitung vom 20. November 2012. Abgerufen am 8. August 2018 von http://www.faz.net/aktuell/politik/inland/urteil-zum-streikrecht-eine-bestaetigung-fuer-diekirchen-11966698.html

Bischoff, C. (1992). Frauen in der Krankenpflege. Frankfurt am Main/New York: Campus.

Bode, I. (2010). Die Malaise der Krankenhäuser. Leviathan, 38, 189-211. doi: 10.1007/s11578-010-0082-x

Braun, M. (2015). Ludwigsburg: Streit am Krankenhaus eskaliert. Stuttgarter Zeitung vom 9. Juni 2015. Abgerufen am 2. Mai 2018 von https://www.stuttgarter-zeitung.de/inhalt.ludwigsburg-streit-am-krankenhaus-eskaliert.3a67482345dc-455e-acc2-e1309f9b5162.html

Bräutigam, C., Evans, M., Hilbert, J. \& Öz, F. (2014). Arbeitsreport Krankenhaus. Eine Online-Befragung von Beschäftigten deutscher Krankenhäuser. Arbeitspapier 306. Düsseldorf: Hans-BöcklerStiftung.

Brinkmann, U. (2011). Die unsichtbare Faust des Marktes: betriebliche Kontrolle und Koordination im Finanzmarktkapitalismus. Berlin: edition Sigma.

Brinkmann, U. \& Nachtwey, O. (2010). Krise und strategische Neuorientierung der Gewerkschaften. Aus Politik und Zeitgeschichte, 13-14/2010, 21-29.

Brinkmann, U., Choi, H.-L., Detje, R., Dörre, K., Holst, H., Karakayali, S. \& Schmalstieg, C. (2008). Strategic Unionism: Aus der Krise zur Erneuerung? Umrisse eines Forschungsprogramms. Wiesbaden: VS Verlag für Sozialwissenschaften.

Brookes, M. (2018). Power Resources in Theory and Practice: Where to go from here. Global Labour Journal 9 (2), 2018, 254-257.

Cropley, A. J. (2002). Qualitative Forschungsmethoden - eine praxisnahe Einführung. Eschborn: Klotz.

Deutscher Berufsverband für Pflegeberufe (DBfK). (2016). Mein Recht auf Frei. Abgerufen am 05. Dezember 2017 von https://www.dbfk.de/media/docs/download/Allgemein/Mein-Recht-auf-Frei_Sammelband-2016.pdf

Dörre, K. (2011). Funktionswandel der Gewerkschaften. Von der intermediären zur fraktalen Organisation. In T. Haipeter \& K. Dörre (Hrsg.), Gewerkschaftliche Modernisierung (S. 267-301). Wiesbaden: VS Verlag für Sozialwissenschaften.

Durkheim, E. (1992 [1893]). Über soziale Arbeitsteilung. Studie über die Organisation höherer Gesellschaften. Frankfurt/Main: Suhrkamp.

Ebbinghaus, B. \& Visser, J. (1999). When Institutions Matter. Union Growth and Decline in Western Europe, 1950-1995. European Sociological Review, 15 (2), 135-158. doi: 10.1093/oxfordjournals.esr.a018257

Folbre, N. (2001). The Invisible Heart: Economics and Family Values. New York: New Press.

Hartfiel, G. \& Hillmann, K.-H. (1972). Wörterbuch der Soziologie. Stuttgart: Kröner.

Haug, W.F. (2009). Gewerkschaften im High-Tech-Kapitalismus vor der Hegemoniefrage. Das Argument, 51 (6), 879-893.

Hechter, M. (1988). Principles of Group Solidarity. Berkeley: University of California Press. 
Hipp, L. \& Kelle, N. (2015). Nur Luft und Liebe? Die Entlohnung sozialer Dienstleistungsarbeit im Länder- und Berufsvergleich. Friedrich-Ebert-Stiftung. Forum Politik und Gesellschaft. Berlin: FES.

Hirsch-Kreinsen, H. (2010). Entgrenzung von Unternehmen und Arbeit. In C. Deutschmann \& J. Beckert (Hrsg.), Wirtschaftssoziologie (S. 447-465). Wiesbaden: VS Verlag.

Honneth, A. (1992). Kampf um Anerkennung. Frankfurt am Main: Suhrkamp.

Hürtgen, S. (2013). Mensch sein auf der Arbeit? Kollegialität als Balance von allgemein-menschlichen und leistungsbezogenen Aspekten von Arbeit. In L. Billmann \& J. Held (Hrsg.). Solidarität in der Krise: Gesellschaftliche, soziale und individuelle Voraussetzungen solidarischer Praxis (S. 237-262). Tübingen: Springer VS.

Kirch, D. (2018). Verdi droht mit langem Streik an Uniklinik Homburg. Saarbrücker Zeitung vom 8. Juni 2018. Abgerufen am 27. August 2018 von

https://www.saarbruecker-zeitung.de/saarland/saarland/verdi-droht-mit-langem-streik-anuniklinik-homburg_aid-23316423

Koalitionsvertrag (2018). Ein neuer Aufbruch für Europa. Eine neue Dynamik für Deutschland. Ein neuer Zusammenhalt für unser Land. Berlin: CDU, CSU, SPD.

Kröll, T. (2013). Arbeitnehmersolidarität im neoliberalen Strukturwandel. In L. Billmann \& J. Held (Hrsg.). Solidarität in der Krise: Gesellschaftliche, soziale und individuelle Voraussetzungen solidarischer Praxis (S. 78-97). Tübingen: Springer VS.

Lamnek, S. (2005). Qualitative Sozialforschung. Lehrbuch. Weinheim/Basel: Beltz.

Marrs, K. (2007). Ökonomisierung gelungen, Pflegekräfte wohlauf? WSI-Mitteilungen, 9/2007, 502507. doi: 10.5771/0342-300x-2007-9-502

Marx, K. (1972 [1867]). Instruktionen für die Delegierten des Provisorischen Zentralrats zu den einzelnen Fragen. In Marx Engels Werke (MEW) (Bd. 16, S. 196), Berlin: Dietz.

Mayring, P. (2002). Einführung in die qualitative Sozialforschung. Eine Anleitung zu qualitativem Denken. Weinheim und Basel: Beltz.

Mayring, P. (1994). Qualitative Inhaltsanalyse. In A. Boehm, A. Mengel \& T. Muhr (Hrsg.), Texte verstehen (S. 159-175). Konstanz: Universitätsverlag Konstanz.

Müller-Jentsch, W. (1997). Soziologie der industriellen Beziehungen - Eine Einführung. Frankfurt/Main: Campus.

Peters, K. \& Sauer, D. (2005) Indirekte Steuerung - eine neue Herrschaftsform. Zur revolutionären Qualität des gegenwärtigen Umbruchprozesses. In H. Wagner (Hrsg.), „Rentier’ ich mich noch?“ Neue Steuerungskonzepte im Betrieb (S. 23-58). Hamburg: VSA.

Rieder, K. (1999). Zwischen Lohnarbeit und Liebesdienst: Belastungen in der Krankenpflege. Weinheim/München: Juventa.

Rosenbrock, R. \& Gerlinger, T. (2014). Gesundheitspolitik. Eine systematische Einführung. Bern: Huber.

Roth, I. (2011). Die Arbeitsbedingungen in Krankenhäusern aus Sicht der Beschäftigten. Ein Branchenbericht auf Basis des DGB-Index Gute Arbeit. Berlin: ver.di Bereich Innovation und Gute Arbeit.

Scherr, A. (2013). Solidarität im Postmodernen Kapitalismus. In L. Billmann \& J. Held (Hrsg.), Solidarität in der Krise: Gesellschaftliche, soziale und individuelle Voraussetzungen solidarischer Praxis (S. 263-270). Tübingen: Springer VS.

Schmalz, S., Ludwig, C. \& Webster, E. (2018). The Power Resources Approach: Developments and Challenges. Global Labour Journal 9(2), 2018, 113-134. doi: 10.15173/glj.v9i2.3569

Senghaas-Knobloch, E. (2008). Zeit für fürsorgliche Praxis. Pflegeethos und Erfahrungen von Frauen und Männern in Pflegeberufen. In E. Senghaas-Knobloch \& C. Kumbruck (Hrsg.), Vom Liebesdienst zur liebevollen Pflege (S. 77-94). Rehburg-Loccum: Evangelische Akademie Loccum.

Siemens, S. \& Frenzel, M. (2016). Das unternehmerische Wir. Formen der Indirekten Steuerung in Unternehmen. Hamburg: VSA. 
Silver, B. J. (2005). Forces of Labor. Arbeiterbewegungen und Globalisierung seit 1870. Berlin/Hamburg: Assoziation A.

Simon, K. (2018). Was der Streik an der Uniklinik für die Patienten bedeutet. Artikel vom 3. August 2018 bei WAZ-Online. Abgerufen am 8. August 2018 von https://www.waz.de/staedte/essen/was-der-streik-an-der-uniklinik-fuer-die-patienten-bedeutetid215006567.html

Simon, M. \& Mehmecke, S. (2017). Nurse-to-Patient Ratios: Ein internationaler Überblick über staatliche Vorgaben zu einer Mindestbesetzung im Pflegedienst der Krankenhäuser. Working Paper 27. Düsseldorf: Hans-Böckler-Stiftung.

Simon, M. (2016). Die ökonomischen und strukturellen Veränderungen des Krankenhausbereichs seit den 1970er Jahren. In I. Bode \& W. Vogd (Hrsg.), Mutationen des Krankenhauses (S. 29-45). Wiesbaden: Springer.

Simon, M. (2013). Das DRG-System. Grundsätzliche Konstruktionsfehler. Deutsches Ärzteblatt, 39, $1782-1786$.

SpiegelOnline (2018). Fachkräftemangel: Deutschland fehlen mehr als 25.000 Pfleger. Artikel vom 25. April 2018. Abgerufen am 8. August 2018 von http://www.spiegel.de/wirtschaft/soziales/pflege-deutschland-fehlen-mehr-als-25-000fachkraefte-a-1204648.html

Statistisches Bundesamt (2018). Grunddaten der Krankenhäuser 2016, Fachserie 12 Reihe 6.1.1.

Streeck, W. (1979). Gewerkschaften als Mitgliederverbände. Probleme gewerkschaftlicher Mitgliederrekrutierung. In J. Bergmann (Hrsg.), Beiträge zur Soziologie der Gewerkschaften (S. 72110). Frankfurt/Main: Suhrkamp.

Supe, J. (25. Januar 2018). Die Drohung, normal zu arbeiten. junge Welt, 5.

Tenfeld, K. (1998). Arbeiterschaft, Solidarität du Arbeiterbewegung. In K. Bayertz (Hrsg.), Solidarität - Begriff und Problem (S. 195-201). Frankfurt/Main: Suhrkamp.

Thome, H. (1998). Soziologie und Solidarität: Theoretische Perspektiven für die empirische Forschung. In K. Bayertz (Hrsg.), Solidarität - Begriff und Problem (S. 217-262). Frankfurt/Main: Suhrkamp.

Tranow, U. (2012). Das Konzept der Solidarität. Düsseldorf: Springer VS.

Urban, H.-J. (2010). Wohlfahrtsstaat und Gewerkschaftsmacht im Finanzmarkt-Kapitalismus: Der deutsche Fall. WSI-Mitteilungen, 9/2010, 443-450. doi: 10.5771/0342-300X-2010-9-443

Van Parijs, Ph. (1996). Refondé la Solidarité. Paris: Editions du Cerf.

Van Parijs, Ph. (1995). Sauver la Solidarité. Paris: Editions du Cerf.

ver.di (2018a). Celenus taucht ab. Abgerufen am 8. August 2018 von https://gesundheit-soziales.verdi.de/mein-arbeitsplatz/reha-einrichtungen/++co++c7d71a107635-11e8-972a-525400f67940

ver.di (2018b). Entlastung oder Streik. Abgerufen am 13. August 2018 von https://gesundheit-soziales.verdi.de/themen/entlastung/++co++a78f50f6-755e-11e8-b3f9$525400 \mathrm{ff} 2 \mathrm{~b} 0 \mathrm{e}$

ver.di (2018c). Mehr von uns ist besser für alle. Abgerufen am 13. August 2018 von https://gesundheit-soziales.verdi.de/themen/entlastung/++co++3991a0d2-3be8-11e7-bd01$525400423 \mathrm{e} 78$

ver.di (2017a). Mach' mal Pause. Abgerufen am 02. Mai 2018 von http://gesundheit-soziales.verdi.de/themen/entlastung/++co++dc9740ce-e248-11e6-bc13$525400423 \mathrm{e} 78$

ver.di (2017b). Pflegenotstand: Was auf der Strecke bleibt. Abgerufen am 02. Mai 2018 von http://gesundheit-soziales.verdi.de/themen/mehr-personal/++co++7a9029c2-371b-11e7-91e4$52540066 \mathrm{e} 5 \mathrm{a} 9$ 
ver.di (2017c). Händedesinfektion? Klar, nur wann? Abgerufen am 02. Mai 2018 von

http://gesundheit-soziales.verdi.de/themen/entlastung/++co++b4a07b28-6e05-11e7-8b0a$525400 \mathrm{ff} 2 \mathrm{~b} 0 \mathrm{e}$

ver.di (2017d). Mehr von uns ist besser für alle. Abgerufen am 11. Mai 2018 von

http://gesundheit-soziales.verdi.de/themen/entlastung/++co++3991a0d2-3be8-11e7-bd01$525400423 \mathrm{e} 78$

ver.di (2017e). Union Busting: „Krachend gescheitert“. Abgerufen am 8. August 2018 von

https://gesundheit-soziales.verdi.de/themen/entlastung/++co++0ea8eeb4-dfff-11e7-8a4a$525400423 \mathrm{e} 78$

ver.di (2016). Aktion Überstundenberg. Abgerufen am 05. Dezember 2017 von

https://gesundheit-soziales.verdi.de/themen/mehr-personal/++co++cf611028-c16f-11e6-bf70$525400 \mathrm{ed} 87 \mathrm{ba}$

ver.di (2015). Gegendruck. Hilfe zur Selbsthilfe bei Überlastung und Personalnot. Berlin: ver.diFachbereich Gesundheit, Soziale Dienste, Wohlfahrt und Kirchen.

ver.di b+b (2017). Überlastungsanzeige. Abgerufen am 02. Mai 2018 von https://www.verdi-bub.de/service/praxistipps/archiv/ueberlastungsanzeige

ver.di Berlin-Brandenburg. (o. J.). Mehr von uns ist besser für alle. Der Kampf um Entlastung und Gesundheitsschutz an der Berliner Charité. Berlin: ver.di-Landesbezirk Berlin-Brandenburg.

ver.di Region Saar-Trier. (o. J.). Die Ultimaten in der ver.di-Region Saar-Trier. Saarbrücken: ver.diFachbereich 03 der ver.di-Region Saar-Trier.

Visser, J. (1995). Trade unions from a comparative perspective. In J. Van Ruysseveldt, R. Huiskamp, \& J. Hoof (Hrsg.), Comparative industrial and employment relations (S. 37-67). London, Sage.

Vogd, W. (2006). Die Organisation Krankenhaus im Wandel. Eine dokumentarische Evaluation aus Sicht der ärztlichen Akteure. Bern: Huber.

Voges, W. (2002). Pflege alter Menschen als Beruf: Soziologie eines Tätigkeitsfeldes. Wiesbaden: Westdeutscher Verlag.

Wildt, A. (1998). Solidarität - Begriffsgeschichte und Definition heute. In K. Bayertz (Hrsg.), Solidarität - Begriff und Problem (S. 202-216). Frankfurt/Main: Suhrkamp.

Windisch, W. (2017). »Wir haben es selbst in der Hand, noch stärker zu werden!« Die Bewegung für einen Tarifvertrag Entlastung in den saarländischen Krankenhäusern. In I. Artus, P. Birke, S. Kerber-Clasen \& W. Menz (Hrsg.), Sorge-Kämpfe, Auseinandersetzungen um Arbeit in sozialen Dienstleistungen (S. 130-153). Hamburg: VSA.

Wright, E. O. (2000). Working Class Power, Capitalist Class Interests, and Class Compromise. American Journal of Sociology, 105 (4), 957-1002. doi: 10.1086/210397 


\section{DuEPublico}

Duisburg-Essen Publications online
UNIVERSITÄT

$D_{E} U_{S} I_{S} S_{N} \mathbf{U} R$ G

offen im Denken

Ub| $\begin{aligned} & \text { universitäts } \\ & \text { bibliothek }\end{aligned}$

Dieser Text wird via DuEPublico, dem Dokumenten- und Publikationsserver der Universität Duisburg-Essen, zur Verfügung gestellt. Die hier veröffentlichte Version der E-Publikation kann von einer eventuell ebenfalls veröffentlichten Verlagsversion abweichen.

DOI: $\quad 10.3224 /$ indbez.v25i4.05

URN: urn:nbn:de:hbz:464-20210414-102940-5 